\title{
Theory of capillary-induced interactions beyond the superposition approximation
}

Cite as: J. Chem. Phys. 127, 204706 (2007); https://doi.org/10.1063/1.2781420

Submitted: 01 June 2007 . Accepted: 16 August 2007 . Published Online: 27 November 2007

Alvaro Domínguez, Martin Oettel, and S. Dietrich

\section{ARTICLES YOU MAY BE INTERESTED IN}

Force balance of particles trapped at fluid interfaces

The Journal of Chemical Physics 128, 114904 (2008); https://doi.org/10.1063/1.2890035

The "Cheerios effect"

American Journal of Physics 73, 817 (2005); https://doi.org/10.1119/1.1898523

The equilibrium shape of fluid-fluid interfaces: Derivation and a new numerical method for Young's and Young-Laplace equations

The Journal of Chemical Physics 141, 244702 (2014); https://doi.org/10.1063/1.4904391

\section{Lock-in Amplifiers up to $600 \mathrm{MHz}$}
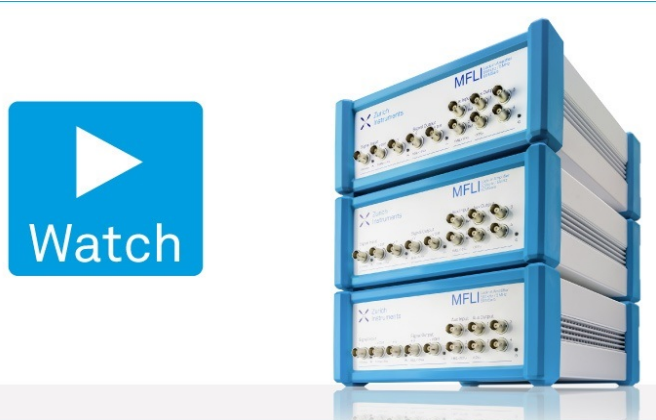

J. Chem. Phys. 127, 204706 (2007); https://doi.org/10.1063/1.2781420 


\title{
Theory of capillary-induced interactions beyond the superposition approximation
}

\author{
Alvaro Domínguez \\ Física Teórica, Universidad de Sevilla, Apartado 1065, E-41080 Sevilla, Spain \\ Martin Oettel \\ Institut für Physik, Universität Mainz, WA 331, D-55099 Mainz, Germany \\ S. Dietrich \\ Max-Planck-Institut für Metallforschung, Heisenbergstrasse 3, D-70569 Stuttgart, Germany and \\ Institut für Theoretische und Angewandte Physik, Universität Stuttgart, Pfaffenwaldring 57, \\ D-70569 Stuttgart, Germany
}

(Received 1 June 2007; accepted 16 August 2007; published online 27 November 2007)

\begin{abstract}
Within a general theoretical framework, we study the effective, deformation-induced interaction between two colloidal particles trapped at a fluid interface in the regime of small deformations. In many studies, this interaction has been computed with the ansatz that the actual interface configuration for the pair is given by the linear superposition of the interface deformations around the single particles. Here, we assess the validity of this approach and compute the leading term of the effective interaction for a large interparticle separation beyond this so-called superposition approximation. As an application, we consider the experimentally relevant case of interface deformations owing to the electrostatic field emanating from charged colloidal particles. In mechanical isolation, i.e., if the net force acting on the total system consisting of the particles plus the interface vanishes, the superposition approximation is actually invalid. The effective capillary interaction is governed by contributions beyond this approximation and turns out to be attractive. For sufficiently small surface charges on the colloids, such that linearization is strictly valid, and at asymptotically large separations, the effective interaction does not overcome the direct electrostatic repulsion between the colloidal particles. (C) 2007 American Institute of Physics.
\end{abstract}

[DOI: $10.1063 / 1.2781420]$

\section{INTRODUCTION}

The self-assembly of submicrometer colloidal particles at fluid (e.g., water/air or water/oil) interfaces has gained significant interest in view of various basic and applied issues such as the study of two-dimensional melting, ${ }^{1}$ investigations of mesoscale structure formation, ${ }^{2}$ and engineering of colloidal crystals on spherical surfaces. ${ }^{3}$ The colloidal particles are trapped at the interface if the fluid phases wet the colloid only partially; this configuration is stable against thermal fluctuations, and it appears to be even the global equilibrium state, in accordance with the experimental observation that the colloids immersed in the bulk phases are attracted toward the interface. ${ }^{1}$

In order to prevent coagulation, the colloidal particles are electrically charged. The ensuing repulsive force is well understood, and at large separations $d$ it varies like a dipoledipole interaction $\propto 1 / d^{4}$ because the monopoles vanish due to screening by counterions in water. ${ }^{4,5}$ Nevertheless, several experimental findings have led to postulating an attractive effective force between such particles with a range much larger than that of van der Waals forces ${ }^{6-15}$ (but see also Ref. 16). Spherical particles (radii $R=0.25-2.5 \mu \mathrm{m}$ ) at flat waterair interfaces exhibit the spontaneous formation of complicated metastable mesostructures consistent with the presence of a minimum in the effective intercolloidal potential at separations $d / R \approx 3-20$ and with a depth of at least a few $k_{B} T$.
Until now, no unequivocal explanation for the appearance of these relatively long-ranged attractions is available. One possibility, which has been explored intensively in previous years, consists of an attraction mediated by the deformation of the interface (see Fig. 1). This is similar to the so-called flotation force attracting particles floating at the surface of water, which is deformed by the weight of the particles. ${ }^{17,18}$ However, gravity plays no role for micrometer sized particles as described above. Instead, the electrostatic field around the charged colloids deforms the interface and gives rise to effective, capillary-induced interactions. There have been contradictory results about the properties of this effective interaction. In Refs. 12 and 19, it has been argued

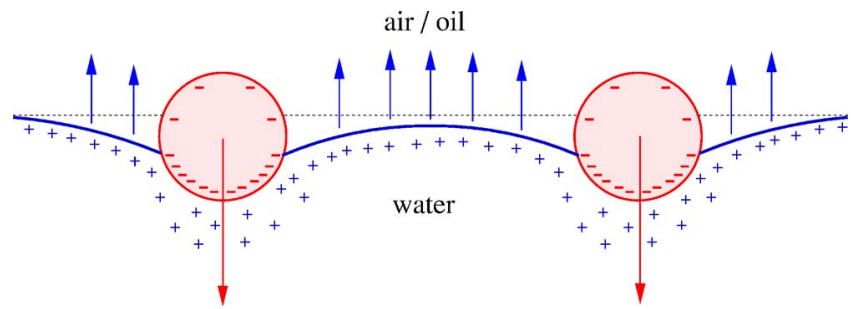

FIG. 1. (Color online) Schematic drawing of the deformation of a fluid interface by electric fields due to charged colloidal particles trapped at the interface. Counterions gather on the side of the electrolytic phase (water), and a pressure field arises, which pulls on the interface and on the particles. 
in favor of an attractive force decaying like $1 / d$ (similar to the gravity-induced flotation force). In Refs. 20-24, this line of thought was shown to be invalid because the decay turns out to be much faster if the physical system consisting of the colloidal particles plus the interface is mechanically isolated, i.e., if the total force acting on this system vanishes in the limit of a macroscopically extended interface with negligible borders. This is, in principle, the case for the experiments conducted in Langmuir troughs with lateral extensions several times the capillary length of the interface (i.e., orders of magnitude larger than the colloid radius). In this case, the electrostatic force pulling on the interface is counterbalanced exactly by the electrostatic force pushing the particles into water. In particular, the authors of Ref. 20 argue for an attractive force decaying like $1 / d^{7}$. However, this was, in turn, corrected in Refs. 21 and 22, and a repulsive force with an asymptotic decay $\propto 1 / d^{7}$ was derived. In Ref. 22 , it was noticed that this latter result is actually unreliable because the linear superposition approximation employed in these calculations is not valid if mechanical isolation holds. Furthermore, since it is the electric field $\mathbf{E}$, and not the electrostatic pressure $\propto \mathbf{E}^{2}$, which obeys a superposition principle, the superposition approximation will be unsuitable if the main contribution to the electrostatic pressure stems from cross terms in $\mathbf{E}^{2}$. This latter case was studied in Refs. 25 and 26, with the conclusion that the capillary-induced force is attractive and decays like $1 / d^{4}$, which is the same asymptotic behavior as the direct dipole-dipole repulsion; whether the capillary attraction overcomes the electric repulsion must then be determined by a detailed analysis of the electrostatic problem. The total force is repulsive in the regime of small deformations of a flat interface (equivalent to the regime of small colloidal charges), for which the calculations were carried out. $^{25,26}$ Only for sufficiently large colloidal charges, the capillary attraction may asymptotically overcome the electrostatic repulsion, leading to a minimum in the total effective potential. ${ }^{25}$ However, further calculations-going beyond the linearization assumptions in treating the electrostatic pressure on the interface and the energy of the deformed interface - are necessary to substantiate this claim. The experiment described in Ref. 12 is peculiar in the sense that the unperturbed interface is actually that of a relatively small spherical droplet pending from a plate. The importance of this finite-size effect was studied in Refs. 22, 23, and 27-29. In Refs. 23, 27, and 29, it was found that the flotationlike decay $1 / d$ is present because the plate breaks the condition of mechanical isolation, but it is quantitatively too small to explain the experimental observations. ${ }^{30}$

Here, we follow the approach of Ref. 22 in order to calculate the capillary-induced effective interaction beyond the superposition approximation in the case that there is a pressure field of the general form $\hat{\Pi}(\mathbf{r})$ acting on the interface in the limit of small deformations. The limit of small deformations corresponds to an analysis to leading order in the small dimensionless parameters $\hat{\varepsilon}_{F}$ and $\hat{\varepsilon}_{\Pi}$, defined in Eq. (1) below, which are measures of the force acting on the colloidal particles and the interface, respectively. In this manner, inter alia, we provide a mathematically sound derivation of the results reported in Refs. 25 and 26. In Sec. II,

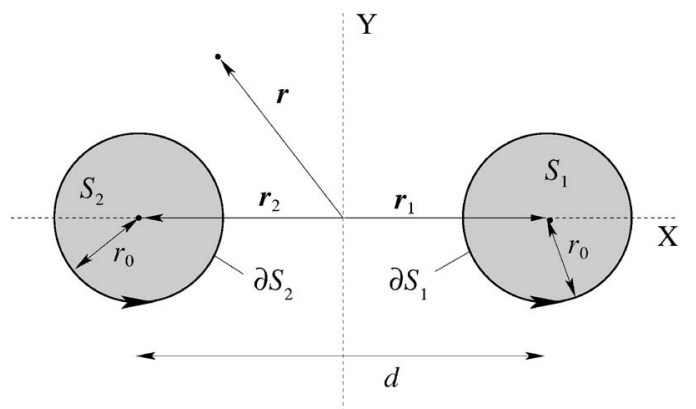

FIG. 2. Top view (plane $z=0$ ) of the reference configuration with two colloids. $d$ is the fixed lateral distance between the colloid centers projected onto the plane $z=0 . S_{1}$ and $S_{2}$ are disks of radius $r_{0}$, the corresponding circumferences (counterclockwise) are $\partial S_{1}$ and $\partial S_{2}$. The (projected) interface is $\hat{S}_{\text {men }}=\mathbb{R}^{2} \backslash\left(S_{1} \cup S_{2}\right)$. The position of any point on the plane is denoted with $\mathbf{r}$; in particular, $\mathbf{r}_{\alpha}$ is the position of the colloid $\alpha$.

we derive the exact leading-order expressions for the capillary potential and show in which respect the superposition approximation becomes inconsistent if mechanical isolation holds. In such a case, the final result depends on the stage at which the approximation is introduced and applied. In Sec. II $\mathrm{B}$, we derive the leading asymptotic dependence on $d$ of the effective interaction using only some rather general assumptions on the form of $\hat{\Pi}(\mathbf{r})$. The result for the effective potential energy is summarized in Eq. (27). In Sec. III, we analyze the particular case that the pressure field $\hat{\Pi}(\mathbf{r})$ is due to the electric field created by charged colloidal particles in a mechanically isolated system. We consider two limiting cases in order to solve the electrostatic problem: (i) water as a perfect conductor (i.e., vanishing Debye length) and (ii) the colloidal particles as pointlike objects (i.e., vanishing particle radius). In both cases, we recover the conclusion that the effective interaction is attractive but can overcome the electric repulsion only if $\hat{\varepsilon}_{F} \gtrsim 1$, which is outside the smalldeformation regime considered here. Finally, in Sec. IV, we summarize our results and discuss their relevance in connection with the experiments described in the literature.

\section{FREE ENERGY OF EFFECTIVE CAPILLARY INTERACTION}

\section{A. Exact results}

We consider $N$ identical $^{31}$ spherical particles trapped at a fluid interface (see Fig. 2 for $N=2$ ). We define the reference configuration as a flat interface in the plane $z=0$ and the colloids at a height such that the colloid-interface contact occurs at Young's contact angle $\theta \in(0, \pi)$. In this configuration, $S_{\alpha}$ denotes the circular disk delimited by the contact line on the colloid $\alpha, \partial S_{\alpha}$ is the corresponding contact line (of radius $r_{0}=R \sin \theta$ for a particle of radius $R$ ) traced counterclockwise when viewed from the top, ${ }^{32}$ and $\hat{S}_{\text {men }}$ is the fluid interface (with surface tension $\gamma$ ), enclosed by a boundary $C_{L}$ of typical size of the order of $L$ representing, e.g., the vessel containing the system. The relative lateral positions of the colloids are kept fixed, and thus we consider only vertical displacements $\hat{u}(\mathbf{r}=(x, y))$ of the fluid interface and of the height $\Delta \hat{h}_{\alpha}$ of the center of the colloid $\alpha$ relative to the plane $z=0$. In the reference configuration, there is a (vertical) force 
$\hat{F}_{\alpha}$ acting on the colloid $\alpha$ and a (vertical) force per unit area $\hat{\Pi}(\mathbf{r})$ on the meniscus. We define the dimensionless forces

$$
\hat{\varepsilon}_{F_{\alpha}}:=-\frac{\hat{F}_{\alpha}}{2 \pi \gamma r_{0}}, \quad \hat{\varepsilon}_{\Pi}:=\frac{1}{2 \pi \gamma r_{0}} \int_{\hat{S}_{\text {men }}} d A \hat{\Pi},
$$

where $d A$ is the element of interface area. The reference configuration is the equilibrium state in the absence of forces, $\hat{F}_{\alpha} \equiv 0, \hat{\Pi} \equiv 0$. Within the approximation of small deviations from the reference configuration, the free energy of the system with respect to this configuration is ${ }^{22}$

$$
\begin{aligned}
\hat{\mathcal{F}}= & \gamma \int_{\hat{S}_{\text {men }}} d A\left[\frac{1}{2}|\nabla \hat{u}|^{2}-\frac{1}{\gamma} \hat{\Pi} \hat{u}\right] \\
& +\sum_{\alpha=1}^{N}\left\{\frac{\gamma}{2 r_{0}} \oint_{\partial S_{\alpha}} d \ell\left[\Delta \hat{h}_{\alpha}-\hat{u}\right]^{2}-\hat{F}_{\alpha} \Delta \hat{h}_{\alpha}\right\}+\mathcal{O}\left(\hat{\varepsilon}_{F}, \hat{\varepsilon}_{\Pi}\right)^{3},
\end{aligned}
$$

where $d \ell$ is the element of arclength. The free energy contains a contribution from the change of the contact area between the phases (two fluid phases and the solid particles) and a contribution from the work done by the forces $\hat{F}_{\alpha}$ and $\hat{\Pi}$ via displacements from the reference configuration.

The values of $\hat{u}(\mathbf{r})$ and $\Delta \hat{h}_{\alpha}$ in the equilibrium state are determined by minimizing this free energy. This leads to the following equations:

$$
\begin{aligned}
& \Delta \hat{h}_{\alpha}=\langle\hat{u}\rangle_{\alpha}-r_{0} \hat{\varepsilon}_{F_{\alpha}}, \quad\langle\cdot\rangle_{\alpha}:=\frac{1}{2 \pi r_{0}} \oint_{\partial S_{\alpha}} d \ell(\cdot), \\
& \nabla^{2} \hat{u}=-\frac{1}{\gamma} \hat{\Pi}, \quad \mathbf{r} \in \hat{S}_{\mathrm{men}}, \\
& \mathbf{n}_{\alpha} \cdot \nabla \hat{u}(\mathbf{r})=\hat{\varepsilon}_{F_{\alpha}}+\frac{\hat{u}(\mathbf{r})-\langle\hat{u}\rangle_{\alpha}}{r_{0}}, \quad \mathbf{r} \in \partial S_{\alpha}, \\
& \hat{u}(\mathbf{r})=0, \quad \mathbf{r} \in C_{L},
\end{aligned}
$$

where $\mathbf{n}_{\alpha}$ is the unit vector in the outward normal direction of $\partial S_{\alpha}$. Equation (3a) is a geometrical relationship, Eq. (3b) describes a local mechanical equilibrium (the pressure $\hat{\Pi}$ is compensated by the curvature-induced interfacial tension), Eq. (3c) describes the mechanical equilibrium of the particle (the force $\hat{F}_{\alpha}$ is balanced by the interfacial tension exerted at the contact line), and Eq. (3d) represents a boundary condition at the external border $C_{L}$ (for simplicity we take a pinned interface, but, of course, other physically reasonable boundary conditions are possible, the details of which are actually irrelevant in the limit $L \rightarrow \infty$ we shall consider $^{22}$ ). The free energy functional in Eq. (2) evaluated at the equilibrium configuration can be simplified by using the relationships in Eqs. (3a)-(3d), and we obtain two equivalent expressions:

$$
\hat{\mathcal{F}}_{\text {eq }}=-\frac{1}{2} \gamma \int_{\hat{S}_{\text {men }}} d A|\nabla \hat{u}|^{2}+\pi \gamma \sum_{\alpha=1}^{N}\left[\langle\hat{u}\rangle_{\alpha}^{2}-\left\langle\hat{u}^{2}\right\rangle_{\alpha}-r_{0}^{2} \hat{\varepsilon}_{F_{\alpha}}^{2}\right]
$$

$$
=-\frac{1}{2} \int_{\hat{S}_{\text {men }}} d A \hat{\Pi} \hat{u}+\pi \gamma \sum_{\alpha=1}^{N} r_{0} \hat{\varepsilon}_{F_{\alpha}}\left[\langle\hat{u}\rangle_{\alpha}-r_{0} \hat{\varepsilon}_{F}\right] .
$$

From this point onward, we shall consider the particular case of two identical colloids. We assume that any external force acting on the system (e.g., gravity) is independent of the positions of the particles, ${ }^{33}$ so that symmetry arguments will allow one to simplify the expressions. We use the notations $u_{\alpha}(\mathbf{r}):=u\left(\left|\mathbf{r}-\mathbf{r}_{\alpha}\right|\right), \Pi_{\alpha}(\mathbf{r}):=\Pi\left(\left|\mathbf{r}-\mathbf{r}_{\alpha}\right|\right), \quad \varepsilon_{F}:=\hat{\varepsilon}_{F_{\alpha}}$, and $S_{\text {men, } \alpha}:=\mathbb{R}^{2} \backslash S_{\alpha}$ for the corresponding quantities in the presence of a single colloid located at position $\mathbf{r}_{\alpha}$. This means that the function $u_{\alpha}(\mathbf{r})(\alpha=1,2)$ satisfies

$$
\begin{aligned}
& \nabla^{2} u_{\alpha}=-\frac{1}{\gamma} \Pi_{\alpha}, \quad \mathbf{r} \in S_{\mathrm{men}, \alpha}, \\
& \mathbf{n}_{\alpha} \cdot \nabla u_{\alpha}(\mathbf{r})=\varepsilon_{F}, \quad \mathbf{r} \in \partial S_{\alpha}, \\
& u_{\alpha}(\mathbf{r})=0, \quad \mathbf{r} \in C_{L} .
\end{aligned}
$$

In terms of these single-colloid solutions, the configuration in the presence of two colloids can be written without loss of generality as

$$
\begin{aligned}
& \hat{\Pi}=\Pi_{1}+\Pi_{2}+2 \Pi_{m}, \\
& \hat{u}=u_{1}+u_{2}+u_{m}, \\
& \hat{\varepsilon}_{F}=\varepsilon_{F}+\varepsilon_{m},
\end{aligned}
$$

where we have introduced $\hat{\varepsilon}_{F}:=\hat{\varepsilon}_{F_{1}}=\hat{\varepsilon}_{F_{2}}$, reflecting the symmetry of the problem. The fields $u_{m}(\mathbf{r})$ and $\Pi_{m}(\mathbf{r})$, and the quantity $\varepsilon_{m}$ introduced this way represent the corrections to the so-called superposition approximation, which is defined by setting

$$
\begin{aligned}
& u_{m}=0, \\
& \Pi_{m}=0, \\
& \varepsilon_{m}=0,
\end{aligned}
$$

i.e., the effects of other particles on the single-particle configuration are neglected altogether. From Eqs. (3a)-(3d) and (5a)-(5c), one can derive the following equations linking $u_{m}(\mathbf{r}), \Pi_{m}(\mathbf{r})$, and $\varepsilon_{m}$ (with $\left.\alpha, \beta=1,2\right)$ :

$$
\begin{aligned}
& \nabla^{2} u_{m}=-\frac{2}{\gamma} \Pi_{m}, \quad \mathbf{r} \in \hat{S}_{\mathrm{men}}, \\
& \mathbf{n}_{\alpha} \cdot \nabla u_{m}-\frac{u_{m}-\left\langle u_{m}\right\rangle_{\alpha}}{r_{0}}=\varepsilon_{m}-\mathbf{n}_{\alpha} \cdot \nabla u_{\beta}+\frac{u_{\beta}-\left\langle u_{\beta}\right\rangle_{\alpha}}{r_{0}}, \\
& \mathbf{r} \in \partial S_{\alpha} \quad(\beta \neq \alpha), \\
& u_{m}(\mathbf{r})=0, \quad \mathbf{r} \in C_{L} .
\end{aligned}
$$

The superposition approximation is violated even if $\Pi_{m}=0$ and $\varepsilon_{m}=0$ because of the boundary conditions at the contact lines [Eq. (8b)], as pointed out in Ref. 22. The case of nonvanishing $\Pi_{m}$ was addressed in Refs. 25 and 26. 
The capillary-induced effective interaction energy is defined as $V_{\text {men }}(d):=\hat{\mathcal{F}}_{\text {eq }}-2 \mathcal{F}_{\text {eq }}$, where $2 \mathcal{F}_{\text {eq }}$ is the sum of the equilibrium free energies of the single-colloid configurations, i.e., for $d \rightarrow \infty$. $V_{\text {men }}(d)$ depends parametrically on the (lateral) separation $d$ of the colloid centers in the reference configuration. This is not the total interaction potential, which must include, e.g., the direct electrostatic repulsion between charged colloids, not considered in expression (2) for the free energy. The effective interaction energy can be written as $V_{\text {men }}=V_{\text {sup }}+V_{\text {corr }}$, where $V_{\text {sup }}$ is the result of imposing the superposition approximation [Eqs. (7a)-(7c)] and $V_{\text {corr }}$ is the correction to this approximation. If we use expression (4a), we obtain

$$
\begin{aligned}
{ }^{a} V_{\text {sup }}= & -\gamma \int_{\hat{S}_{\text {men }}} d A\left(\nabla u_{1}\right) \cdot\left(\nabla u_{2}\right)+\gamma \int_{S_{1}} d A\left|\nabla u_{2}\right|^{2} \\
& -2 \pi \gamma\left\langle u_{2}^{2}-\left\langle u_{2}\right\rangle_{1}^{2}\right\rangle_{1}, \\
{ }^{a} V_{\text {corr }}= & -\frac{1}{2} \gamma \int_{\hat{S}_{\text {men }}} d A\left(\nabla u_{m}\right) \cdot \nabla\left(u_{m}+4 u_{2}\right) \\
& -2 \pi \gamma\left\langle u_{m}\left(u_{m}+2 u_{2}\right)-\left\langle u_{m}\right\rangle_{1}\left\langle u_{m}+2 u_{2}\right\rangle_{1}\right\rangle_{1} \\
& -2 \pi \gamma r_{0}^{2} \varepsilon_{m}\left(2 \varepsilon_{F}+\varepsilon_{m}\right) .
\end{aligned}
$$

On the other hand, using expression (4b), we obtain

$$
\begin{aligned}
{ }^{b} V_{\text {sup }}= & -\int_{\hat{S}_{\text {men }}} d A \Pi_{1} u_{2}+\int_{S_{1}} d A \Pi_{2} u_{2}+2 \pi \gamma r_{0} \varepsilon_{F}\left\langle u_{2}\right\rangle_{1}, \\
{ }^{b} V_{\text {corr }}= & -\int_{\hat{S}_{\text {men }}} d A\left[\Pi_{2} u_{m}+2 \Pi_{m} u_{2}+\Pi_{m} u_{m}\right] \\
& +2 \pi \gamma r_{0}\left\langle\left(\varepsilon_{F}+\varepsilon_{m}\right) u_{m}+\varepsilon_{m}\left(u_{1}+u_{2}\right)\right\rangle_{1} \\
& -2 \pi \gamma r_{0}^{2} \varepsilon_{m}\left(2 \varepsilon_{F}+\varepsilon_{m}\right) .
\end{aligned}
$$

We emphasize that the two alternative expressions of $V_{\text {sup }}$ and $V_{\text {corr }}$ are not equivalent (but their sum $V_{\text {men }}$ is), and they in turn differ from $V_{\text {sup }}$ as computed in Ref. 22 [see Eq. (39) therein], which was derived by inserting the superposition ansatz directly into Eq. (2) here. The application of Gauss' theorem with Eq. (5a) leads to

$$
\begin{aligned}
{ }^{a} V_{\text {sup }}-{ }^{b} V_{\text {sup }} & =2 \pi \gamma\left\langle r_{0}\left(u_{1}+u_{2}\right) \frac{\partial u_{2}}{\partial n_{1}}-\left(u_{2}-\left\langle u_{2}\right\rangle_{1}\right)^{2}\right\rangle_{1} \\
& ={ }^{b} V_{\text {corr }}-{ }^{a} V_{\text {corr }} .
\end{aligned}
$$

The superposition approximation is inconsistent asymptotically in cases in which ${ }^{a} V_{\text {sup }}-{ }^{b} V_{\text {sup }}$ does not decay more rapidly than ${ }^{a} V_{\text {sup }}$ as a function of the separation $d$. As remarked in Ref. 22, there are indeed relevant cases in which this consistency condition is not fulfilled (see next section).

\section{B. Effective potential in the intermediate asymptotic regime $r_{0} \ll d \ll L$}

In this section, we compute $V_{\text {men }}(d)$ asymptotically in the intermediate range $r_{0} \ll d \ll L$. For this purpose, we have to make some restricting assumptions which, however, seem to be satisfied in the experimental setups investigated so far. First, in view of the discussion in the Introduction concerning the electrical fields, we assume the proportionality

$$
\Pi_{m}^{2} \sim\left|\Pi_{1} \Pi_{2}\right| .
$$

This is valid if the interface stress is quadratic in a field satisfying linear superposition in the two-particle configuration. Examples are given below by some specific electrostatic models [see Eqs. (31) and (49)]. Second, we assume that the single-colloid pressure $\Pi$ decays far from the colloid as

$$
\Pi(r) \sim r^{-n}, \quad n>4 .
$$

In the experimentally relevant case of charged particles at water interface, it has been established both theoretically ${ }^{4}$ and experimentally ${ }^{34}$ that $n=6$. (This can be understood easily: the charge of a particle induces a screening image charge in the water, so that the distant electric field is dipolar.) The constraint $n>4$ will allow us to estimate the integrals appearing in Eqs. (9a), (9b), (10a), and (10b) by approximating them by the contribution of the regions near the colloids. This condition excludes, however, the effect of an external electric field (in that case, the resulting pressure does not have to decay at all) and the case that the colloidal charge is not perfectly screened so that the distant electric field corresponds to a monopole, i.e., $n=4$ (this would occur if both fluid phases are dielectric, e.g., air and insulating oil).

The quantity $\hat{\varepsilon}_{\Pi}-2 \hat{\varepsilon}_{F}$ is the net (vertical) force by an external agent acting on the total system consisting of two colloids plus the interface (see Appendix A). This can be, e.g., gravity (if the colloid is large enough for it to be quantitatively relevant), dispersion forces by a substrate closely beneath the interface (this effect can be modeled similarly as gravity, see Appendix B), or an optical tweezer pushing the colloid vertically. Since we have assumed previously that this external force is independent of the positions of the particles, it is given by the sum of the net forces in the singleparticle configuration (i.e., if they are infinitely far apart from each other):

$$
\hat{\varepsilon}_{\Pi}-2 \hat{\varepsilon}_{F}=2\left(\varepsilon_{\Pi}-\varepsilon_{F}\right) .
$$

From the definitions in Eqs. (1) and (6a), we can write

$$
\hat{\varepsilon}_{\Pi}=2\left(\varepsilon_{\Pi}+\varepsilon_{\Pi_{m}}-\varepsilon_{12}\right),
$$

with

$$
\varepsilon_{\Pi_{m}}:=\frac{1}{2 \pi \gamma r_{0}} \int_{\hat{S}_{\text {men }}} d A \Pi_{m}, \quad \varepsilon_{12}:=\frac{1}{2 \pi \gamma r_{0}} \int_{S_{1}} d A \Pi_{2} .
$$

For $d \rightarrow \infty$, it is clear that

$$
\varepsilon_{12} \sim \frac{1}{d^{n}} .
$$

In this limit, we note that $\varepsilon_{\Pi_{m}}$ receives its main contribution from the regions around $S_{\alpha}$, implying 


$$
\varepsilon_{\Pi_{m}} \sim \frac{2}{\gamma r_{0}} \int_{r_{0}}^{\infty} d r r \Pi_{m}(r) \sim \frac{1}{d^{n / 2}} \int_{r_{0}}^{\infty} d r \frac{r}{r^{n / 2}}
$$

provided $n>4$, because $\left|\Pi_{m}(\mathbf{r})\right| \sim \sqrt{\Pi(d) \Pi\left(\left|\mathbf{r}-\mathbf{r}_{\alpha}\right|\right)}$ in those regions which provide the dominant contribution to the integral. Therefore, from Eqs. (6c) and (14), one obtains asymptotically

$$
\varepsilon_{m}=\varepsilon_{\Pi_{m}}-\varepsilon_{12} \sim \varepsilon_{\Pi_{m}} \sim \frac{1}{d^{n / 2}} .
$$

With these simplifying assumptions, we shall compute analytically the behavior of $V_{\text {men }}(d \rightarrow \infty)$ to leading order in $1 / d$. More precisely, on dimensional grounds ${ }^{35}$ the expansion parameter is $r_{0} / d$. In principle, $\hat{\Pi}$ can contain and thus introduce additional length scales, for example, the Debye length if the fluid phase is an electrolyte. This complicates the problem, which then has to be analyzed numerically (see Sec. III B).

\section{The superposition approximation}

Within the superposition approximation, $V_{\text {men }}(d)$ was computed in detail in Ref. 22. Here, we sketch briefly the estimate of the asymptotic behavior of ${ }^{b} V_{\text {sup }}$ [compare the three terms in Eq. (10a)],

$$
\begin{aligned}
\int_{\hat{S}_{\text {men }}} d A \Pi_{1} u_{2} \sim & 2 \pi \gamma r_{0} \varepsilon_{\Pi} u(d)+\Pi(d) \\
\times & \left.\times 2 \int_{S_{\text {men }, 2}} d A u_{2}-\frac{1}{2} \pi r_{0}^{3} \varepsilon_{\Pi}+\pi r_{0}^{2}\left\langle u_{2}\right\rangle_{2}\right],
\end{aligned}
$$

because the main contribution stems from the regions around $S_{\alpha}$, and

$$
\begin{aligned}
& \int_{S_{1}} d A \Pi_{2} u_{2} \sim \Pi(d) u(d) \int_{S_{1}} d A \\
& 2 \pi \gamma r_{0} \varepsilon_{F}\left\langle u_{2}\right\rangle_{1} \sim 2 \pi \gamma r_{0} \varepsilon_{F}\left[u(d)-\frac{1}{4 \gamma} r_{0}^{2} \Pi(d)\right]
\end{aligned}
$$

after expanding around $r_{2}=d$. Thus,

$$
\begin{aligned}
{ }^{b} V_{\text {sup }}(d) \sim & 2 \pi \gamma r_{0}\left(\varepsilon_{F}-\varepsilon_{\Pi}\right) u(d)-\Pi(d) \\
& \times\left[2 \int_{S_{\text {men, } 2}} d A u_{2}+\pi r_{0}^{2}\left\langle u_{2}\right\rangle_{2}\right]
\end{aligned}
$$

because $\Pi(d)$ is asymptotically subdominant compared with $u(d)$ [see Eqs. (13) and (A1)]. The difference ${ }^{a} V_{\text {sup }}-{ }^{b} V_{\text {sup }}$ in Eq. (11) between the two implementations of the superposition approximation can be estimated as ${ }^{22}$ [see Eq. (A1)]

$$
\left\langle\left(u_{1}+u_{2}\right) \frac{\partial u_{2}}{\partial n_{1}}\right\rangle_{1} \approx \frac{1}{2} r_{0}\left\langle u_{1}\right\rangle_{1} \nabla^{2} u(d) \propto \Pi(d) \sim \frac{1}{d^{n}},
$$

$$
\begin{aligned}
\left\langle\left(u_{2}-\left\langle u_{2}\right\rangle_{1}\right)^{2}\right\rangle_{1} & \approx \frac{1}{2} r_{0}^{2}|\nabla u(d)|^{2} \\
& \sim\left\{\begin{array}{l}
1 / d^{2(n-1)} \quad \text { if } \varepsilon_{F}-\varepsilon_{\Pi}=0 \\
1 / d^{2} \quad \text { if } \varepsilon_{F}-\varepsilon_{\Pi} \neq 0 .
\end{array}\right.
\end{aligned}
$$

Thus, whenever $\varepsilon_{F}-\varepsilon_{\Pi} \neq 0$ (i.e., the system is not mechanically isolated in the sense that there must be a force acting on the boundary $C_{L}$ of the interface to compensate this nonvanishing net force), one finds

$$
\begin{aligned}
{ }^{b} V_{\text {sup }}(d) & \sim 2 \pi \gamma r_{0}\left(\varepsilon_{F}-\varepsilon_{\Pi}\right) u(d) \\
& \sim 2 \pi \gamma r_{0}^{2}\left(\varepsilon_{F}-\varepsilon_{\Pi}\right)^{2} \ln \frac{d}{L}+\mathrm{const},
\end{aligned}
$$

corresponding to an attractive force irrespective of the precise form of the function $\Pi(r)$. (The additive constant, which does not affect the physical conclusions, depends on the precise form of the boundary condition at $C_{L}$.) Physically, ${ }^{b} V_{\text {sup }}$ is the work done by the net force $2 \pi \gamma\left(\varepsilon_{F}-\varepsilon_{\Pi}\right)$ upon a vertical shift of the subsystem consisting of one colloid plus its surrounding interface (behaving like an "effective particle") by an amount $u(d)$ due to the deformation induced by the second colloid. In this case (i.e., $\varepsilon_{F}-\varepsilon_{\Pi} \neq 0$ ), the difference ${ }^{a} V_{\text {sup }}-{ }^{b} V_{\text {sup }}$ decays more rapidly than ${ }^{b} V_{\text {sup }}(d)$, and both expressions ${ }^{a} V_{\text {sup }}$ and ${ }^{b} V_{\text {sup }}$ agree asymptotically. Equation (21) exhibits the same dependence on $d$ as the potential energy associated with the flotation force; this is discussed briefly in Appendix B.

If $\varepsilon_{F}-\varepsilon_{\Pi}=0$ (corresponding to mechanical isolation), ${ }^{b} V_{\text {sup }}(d \rightarrow \infty) \sim 1 / d^{n}$ [see Eq. (13)]. This decay agrees with previous findings, ${ }^{20-22}$ but the reliability of this result is unclear because the difference ${ }^{a} V_{\text {sup }}-{ }^{b} V_{\text {sup }}$ decays with the same power law. As a matter of fact, the amplitudes of the asymptotic decay of ${ }^{a} V_{\text {sup }}$ and ${ }^{b} V_{\text {sup }}$ differ and are, in turn, different from Eq. (52) in Ref. 22 because there actually neither of the two representations of $\hat{\mathcal{F}}_{\text {eq }}$ was used. However, for the leading behavior, this is unimportant because $V_{\text {men }}$ is asymptotically dominated by the correction to the superposition approximation.

\section{Beyond the superposition approximation}

If the interface deformation field in Eq. (6b) is evaluated near colloid 1 , the term $u_{2}+u_{m}$ is dominated by $u_{2}$ in the absence of mechanical isolation and by $u_{m}$ in the case of mechanical isolation [see Eqs. (A1) and (A9)],

$$
u_{2}+u_{m} \sim\left\{\begin{array}{c}
u_{m}(d) \sim d^{-n / 2} \quad \text { if } \varepsilon_{F}-\varepsilon_{\Pi}=0 \\
u_{2}(d) \sim \ln d \quad \text { if } \varepsilon_{F}-\varepsilon_{\Pi} \neq 0 .
\end{array}\right.
$$

Thus, the superposition approximation holds if and only if the system is not mechanically isolated. Otherwise, the correction $u_{m}$ is dominant, and for asymptotically large separations $d, V_{\text {men }} \sim V_{\text {corr }}$. Hence, in the following, we take $\varepsilon_{F}$ $-\varepsilon_{\Pi}=0$. Since the main contributions to the integrals stem from the regions around $S_{\alpha}$, one obtains the estimates 


$$
\begin{aligned}
& -\int_{\hat{S}_{\text {men }}} d A\left[\Pi_{2} u_{m}+2 \Pi_{m} u_{2}+\Pi_{m} u_{m}\right] \\
& \quad \sim-\int_{S_{\text {men }, 2}} d A\left[\Pi_{2} u_{m}+2 \Pi_{m} u_{2}\right] \sim \frac{1}{d^{n / 2}} .
\end{aligned}
$$

Using the asymptotic decay of $u_{m}$ given by Eq. (A9), we find

$$
\begin{aligned}
{ }^{b} V_{\text {corr }}(d) \sim & -\int_{S_{\text {men }, 2}} d A\left[\Pi_{2} u_{m}+2 \Pi_{m} u_{2}\right] \\
& +2 \pi \gamma r_{0}\left\langle\varepsilon_{F} u_{m}+\varepsilon_{\Pi_{m}} u_{1}\right\rangle_{1}-4 \pi \gamma r_{0}^{2} \varepsilon_{F} \varepsilon_{\Pi_{m}} \sim \frac{1}{d^{n / 2}}
\end{aligned}
$$

because each term scales like $d^{-n / 2}$ and there is no reason for mutual cancellations. In this expression, one can identify two distinct contributions with a simple physical meaning. After inserting Eqs. (6b) and (6c) into relationship (3a), we write $\Delta \hat{h}_{2}=\Delta h_{2}+\Delta h_{m}$ with

$$
\begin{aligned}
& \Delta h_{2}:=\left\langle u_{1}+u_{2}\right\rangle_{2}-r_{0} \varepsilon_{F} \quad\left(\sim\left\langle u_{2}\right\rangle_{2}-r_{0} \varepsilon_{F}+\mathcal{O}\left(d^{-n}\right)\right), \\
& \Delta h_{m}:=\left\langle u_{m}\right\rangle_{2}-r_{0} \varepsilon_{m} \quad\left(\sim d^{-n / 2}\right),
\end{aligned}
$$

where the asymptotic decays are given by Eqs. (18) and (A9). Accordingly, expression (24) shows that ${ }^{b} V_{\text {corr }}$ is dominated asymptotically by ${ }^{36}$ (i) the work done by the additional pressure $2 \Pi_{m}$ if the single-colloid configuration is deformed relative to the reference configuration, that is, $-\int d A 2 \Pi_{m} u_{2}$ $+2 \pi \gamma r_{0} \varepsilon_{\Pi_{m}} \Delta h_{2}$, and (ii) the work done by the forces acting in the single-colloid configuration upon the additional displacement $u_{m}$, that is, $-\int d A \Pi_{2} u_{m}+2 \pi \gamma r_{0} \varepsilon_{F} \Delta h_{m}$.

The asymptotic behavior of ${ }^{a} V_{\text {corr }}(d \rightarrow \infty)$ can be derived by estimating the behavior of the terms in Eq. (9b) individually, as in the case of Eq. (10b), with the following result:

$$
\begin{aligned}
{ }^{a} V_{\text {corr }}(d) \sim & -2 \gamma \int_{S_{\text {men }, 2}} d A\left(\nabla u_{m}\right) \cdot\left(\nabla u_{2}\right) \\
& -4 \pi \gamma r_{0}^{2} \varepsilon_{F} \varepsilon_{\Pi_{m}} \sim \frac{1}{d^{n / 2}} .
\end{aligned}
$$

The asymptotic decay predicted by Eqs. (24) and (26) must agree both with respect to the decay exponents and the amplitudes because the difference ${ }^{a} V_{\text {sup }}-{ }^{b} V_{\text {sup }}$ decays asymptotically more rapidly [see Eqs. (20a) and (20b) for $\varepsilon_{F}-\varepsilon_{\Pi}$ $=0]$. The sign of the force described by $V_{\text {corr }}(d)$ is not evident from the outset, but in the applications we shall consider later, it turns out to be always attractive.

It is interesting to compare our result with the corresponding one in Ref. 26. After noting the equivalence $u_{m} \leftrightarrow 2 u_{12}$ in the notations, one finds that Eq. (7) in Ref. 26 is identical with the integral term in Eq. (26) here. We obtain an additional term $\propto \varepsilon_{F} \varepsilon_{\Pi_{m}}$ because we treat the contribution by the colloid to the free energy functional in full detail, while in Ref. 26 the point-particle approximation $\left(r_{0} \rightarrow 0\right)$ is used from the outset [compare Eq. (4a) here with Eq. (1) in Ref. 26]. As a consequence, in Ref. 26 our additional term is lost as a singularity of the integral term, which is regularized there by introducing an unknown cutoff length expected to be of the order of $r_{0}$ (see also Refs. 21 and 22). The analysis of realistic models in Sec. III will show that the quantitative contribution of the term ${ }^{\propto} \varepsilon_{F} \varepsilon_{\Pi_{m}}$ to the effective interaction $V_{\text {men }}$ is actually larger than, but proportional to, the contribution from the other term in Eq. (26).

To summarize, the capillary-induced effective interaction between two colloids is given by [compare Eq. (13) defining $n]$

$V_{\text {men }}(d) \sim\left\{\begin{array}{cc}V_{\text {corr }}(d) \sim d^{-n / 2} & \text { if } \varepsilon_{F}-\varepsilon_{\Pi}=0 \\ V_{\text {sup }}(d) \sim \ln d & \text { if } \varepsilon_{F}-\varepsilon_{\Pi} \neq 0\end{array} \quad\left(r_{0} \ll d\right)\right.$.

\section{APPLICATIONS}

In this section, we compute $V_{\text {men }}(d)$ for $\varepsilon_{F}=\varepsilon_{\Pi}$ (mechanical isolation) for different realistic models of $\hat{\Pi}$ derived from the solution of the electrostatic problem within various approximations. We note that the asymptotic decay of $V_{\text {men }}(d)$ is the same as that of the direct electrostatic repulsion, so that these detailed calculations beyond the asymptotic analysis of Sec. II B are necessary in order to be able to address this fine-tuning problem and to determine whether the total force is asymptotically attractive or repulsive.

\section{A. Ideally conducting fluid phase}

The simplest model consists of approximating water by an ideal conductor. Formally, this corresponds to the limit of zero temperature, so that the osmotic pressure of the mobile charges accumulated at the interface vanishes and the Debye length $\kappa^{-1}$ is zero [see Eq. (41)]. In this case, the electric field $\hat{\mathbf{E}}=\hat{E} \mathbf{e}_{z}$ is always normal to the interface and the pressure is given by Maxwell's stress tensor evaluated at the insulating side of the interface (we use Gaussian units),

$$
\hat{\Pi}=\left.\frac{1}{4 \pi} \mathbf{e}_{z} \cdot\left(\epsilon \hat{\mathbf{E}} \hat{\mathbf{E}}-\frac{1}{2} \epsilon \hat{E}^{2} \mathbf{1}\right) \cdot \mathbf{e}_{z}\right|_{z=0^{+}}=\frac{\epsilon_{1}}{8 \pi} \hat{E}^{2}\left(z=0^{+}\right),
$$

where $\epsilon_{1}$ is the dielectric constant of the insulating phase $(z>0)$.

In the present context, the electrostatic problem of a charged sphere partially immersed in a conducting fluid has been solved numerically and semianalytically in Ref. 37 . There, it has been shown that the single-colloid pressure field exhibits an integrable divergence upon approaching the three-phase contact line and that asymptotically it displays the familiar dipole behavior. The following approximate parametrization [Eq. (1.4) in Ref. 37 expressed in terms of our notation] incorporates these properties and is sufficiently accurate for our present purposes: 


$$
\begin{aligned}
& \Pi(r)=\frac{\gamma \varepsilon_{F}}{r_{0}} b(\mu)\left[\frac{r}{r_{0}}-1\right]^{\mu-1}\left[\frac{r}{r_{0}}\right]^{-\mu-5}, \\
& b(\mu):=\frac{1}{6} \mu(\mu+1)(\mu+2)(\mu+3),
\end{aligned}
$$

where $0<\mu<1$ is a fitting parameter, the precise value of which depends on the contact angle $\theta$ and the dielectric constant $\epsilon_{1}$. This expression is normalized so that $\varepsilon_{\Pi}=\varepsilon_{F}$, and it corresponds to an exponent $n=6$ independent of the choice for $\mu$. Therefore, far from a colloid, the single-colloid electric field $|\mathbf{E}|=\sqrt{8 \pi \Pi / \epsilon_{1}}$ is that of a dipole perpendicular to the interface, the strength of which is given by

$$
|p|=\lim _{r \rightarrow \infty} \epsilon_{1} r^{3}|\mathbf{E}(r)|=\epsilon_{1} r_{0}^{3} \sqrt{\frac{8 \pi \gamma \varepsilon_{F} b(\mu)}{\epsilon_{1} r_{0}}} .
$$

In the presence of two colloids, we take $\hat{\mathbf{E}} \approx \mathbf{E}_{1}+\mathbf{E}_{2}$. This approximation allows a simplification of the calculations and should not alter the physical picture significantly. The approximative character is due to possible violations of the electrostatic boundary conditions at the surfaces of the colloidal particles: The additional polarization of colloid 1 induced by $\mathbf{E}_{2}$ will actually lead to an electric field $\hat{\mathbf{E}}=\mathbf{E}_{1}$ $+\mathbf{E}_{2}+\delta \mathbf{E}$ in the neighborhood of colloid 1, with an induced electric field $|\delta \mathbf{E}(\mathbf{r}, d)| \sim \chi(\mathbf{r}) E_{2}(d)$, where the "effective susceptibility" $\chi(\mathbf{r})$ is expected to be at most of order unity. Thus, our conclusions will be qualitatively correct with a quantitative error of a factor of order unity. Under these conditions, the field $\Pi_{m}(\mathbf{r})$ defined by Eq. (6a) is given within this approximation by

$$
\Pi_{m}(\mathbf{r})=\sqrt{\Pi\left(\left|\mathbf{r}-\mathbf{r}_{1}\right|\right) \Pi\left(\left|\mathbf{r}-\mathbf{r}_{2}\right|\right)} .
$$

The integrals in Eq. (26) are computed by using the expressions in Eqs. (A1) and (A9) so that in the limit $r_{0} \ll d$, one obtains (for details, see Appendix C)

$$
\varepsilon_{\Pi_{m}} \approx \frac{4 b(\mu)}{\mu+1} \varepsilon_{F}\left(\frac{r_{0}}{d}\right)^{3}
$$

and

$$
\int_{S_{\mathrm{men}, 2}} d A\left(\nabla u_{m}\right) \cdot\left(\nabla u_{2}\right) \approx \frac{8 \pi b(\mu)}{\mu+1} M(\mu) r_{0}^{2} \varepsilon_{F}^{2}\left(\frac{r_{0}}{d}\right)^{3}
$$

with

$$
\begin{aligned}
M(\mu):= & \frac{1}{8}(\mu+1) b(\mu) \\
& \times \int_{0}^{1} d v v_{2}^{4} F_{1}(1-\mu, 4 ; 5 ; v)_{2} F_{1}\left(\frac{1-\mu}{2}, 1 ; 2 ; v\right)
\end{aligned}
$$

and ${ }_{2} F_{1}(\alpha, \beta ; \gamma ; z)$ is the hypergeometric function (see Sec. 9.1 in Ref. 38). We have checked numerically that $M(\mu)$ $\approx \mu / 5$ with a maximum error of $\approx 1.3 \%$ within the range 0 $\leqslant \mu \leqslant 1$. The final result reads

$$
\begin{aligned}
V_{\mathrm{men}}(d) & \approx{ }^{a} V_{\mathrm{corr}}(d) \\
& \approx-\frac{16 \pi b(\mu)}{\mu+1}[1+M(\mu)] \gamma r_{0}^{2} \varepsilon_{F}^{2}\left(\frac{r_{0}}{d}\right)^{3}
\end{aligned}
$$

This corresponds to an attractive force. We note that the dominant contribution to $V_{\text {men }}(d)$ stems from the term proportional to $\varepsilon_{\Pi_{m}}$ in Eq. (26); the more so the smaller $\mu$ is. Smaller values of $\mu$ correspond to an increasing importance of the electric field near the colloid [see Eq. (29)].

$V_{\text {men }}(d)$ is to be compared with the potential energy due to the direct electrostatic repulsion $V_{\text {rep }}(d)$ of the charged colloids. The potential energy of one dipole in the field of another identical dipole is $p^{2} /\left(\epsilon_{1} d^{3}\right)$ so that $V_{\text {rep }}(d)$ $\approx p^{2} /\left(2 \epsilon_{1} d^{3}\right)$ for large $d$. (One must divide by a factor of 2 because within our model no work is done on the image charge inside the conducting phase forming the dipole.) Collecting the results, we find that the total interaction energy at large separations is given by

$$
\begin{aligned}
V_{\text {total }}(d) & =V_{\text {rep }}(d)+V_{\text {men }}(d) \\
& \approx 4 \pi \gamma r_{0}^{2} \varepsilon_{F} b(\mu)\left(\frac{r_{0}}{d}\right)^{3}\left[1-\frac{4}{\mu+1}[1+M(\mu)] \varepsilon_{F}\right]
\end{aligned}
$$

Hence, we see that the attractive capillary potential is proportional to $\varepsilon_{F}^{2}$ and the direct electrostatic repulsion is proportional to $\varepsilon_{F}$. Thus, in the limit $\varepsilon_{F} \ll 1$, on which our calculations are based, the electrostatic repulsion is always larger than the capillary attraction. The leading-order analysis of this model predicts an attraction only if the charge of the colloid is large enough so that $\varepsilon_{F}=\mathcal{O}(1)$. The critical value above which there is attraction is given by

$$
\varepsilon_{F, \text { crit }}(\mu)=\frac{\mu+1}{4[1+M(\mu)]},
$$

which lies in the range $1 / 4<\varepsilon_{F, \text { crit }}<5 / 12$ for $0<\mu<1$.

\section{B. Finite Debye length}

Consider now the more general case of an upper insulating phase (dielectric constant $\epsilon_{1}$ ) and a lower electrolytic phase (dielectric constant $\epsilon_{2}$, electrolyte concentration $n_{0}$ ) at a finite temperature $T$. The stress tensor acting on the interface is due to the difference of Maxwell's stress tensor just above and below the interface, plus an osmotic pressure $\hat{p}_{\text {osm }}$ by the excess of ions concentrated close to the interface,

$$
\hat{\Pi}=\left.\frac{1}{4 \pi} \mathbf{e}_{z} \cdot\left(\epsilon \hat{\mathbf{E}} \hat{\mathbf{E}}-\frac{1}{2} \epsilon \hat{E}^{2} \mathbf{1}\right) \cdot \mathbf{e}_{z}\right|_{z=0^{-}} ^{z=0^{+}}+\hat{p}_{\mathrm{osm}} \cdot
$$

With $\hat{\Phi}(\mathbf{r})$ denoting the electrostatic potential at the interface, $\hat{E}_{z, \pm}(\mathbf{r}):=\mathbf{e}_{z} \cdot \hat{\mathbf{E}}\left(\mathbf{r}, z=0^{ \pm}\right)$the normal component of the electric field at the interface (with $\epsilon_{1} \hat{E}_{z,+}=\epsilon_{2} \hat{E}_{z,-}$ ), and $\hat{\mathbf{E}}_{\|}(\mathbf{r}, z=0)$ the (continuous) parallel component at the interface, we have 


$$
\left.\frac{1}{4 \pi} \mathbf{e}_{z} \cdot\left(\epsilon \hat{\mathbf{E}} \hat{\mathbf{E}}-\frac{1}{2} \epsilon \hat{E}^{2} \mathbf{1}\right) \cdot \mathbf{e}_{z}\right|_{z=0^{-}} ^{z=0^{+}}=\frac{\epsilon_{2}-\epsilon_{1}}{8 \pi}\left[\frac{\epsilon_{1}}{\epsilon_{2}} \hat{E}_{z,+}^{2}+\hat{\mathbf{E}}_{\|}^{2}\right], \quad \mathcal{I}_{c}(k):=\mathcal{I}_{a}(k)-k \frac{d \mathcal{I}_{a}}{d k}(k) .
$$

with $\Delta n(\mathbf{r})$ as the excess ion concentration at the interface,

$$
\hat{p}_{\text {osm }} \approx k_{B} T \Delta n=n_{0} k_{B} T\left[\exp \left(\frac{e \hat{\Phi}}{k_{B} T}\right)+\exp \left(-\frac{e \hat{\Phi}}{k_{B} T}\right)-2\right],
$$

assuming that the electrolyte is dilute and consists of monovalent ions.

In order to solve the electrostatic problem, we introduce two simplifications: We apply the Debye-Hückel approximation for dilute electrolytes, and we approximate the extended colloid by a point charge $q$ at its center; i.e., we retain only the monopole term of the source of the field. This "monopolar" approximation corresponds formally to the limit of vanishing contact radius, $r_{0} \rightarrow 0$, and it can be expected to provide the correct field at distances from the colloid which are large compared to $r_{0}$. This is, in fact, complementary to the limiting case $\kappa r_{0} \gg 1$ considered in the previous section. From Eq. (39), we find

$$
\hat{p}_{\mathrm{osm}} \approx \frac{\epsilon_{2}}{8 \pi} \kappa^{2} \hat{\Phi}^{2}
$$

where the screening length is given by

$$
\kappa^{-1}=\sqrt{\frac{\epsilon_{2} k_{B} T}{8 \pi n_{0} e^{2}}} .
$$

For pure water, $\epsilon_{2}=81$ and $n_{0} \approx 10^{-7} \mathrm{~mol} / 1$ (that is, $p \mathrm{H}=7$ ) lead to a screening length $\approx 1 \mu \mathrm{m}$ at room temperature.

We consider first the case of a single colloid corresponding to a point charge $q$ located at the flat interface between an insulator and an electrolyte. Proceeding along the lines of, e.g., Refs. 4 and 5, we find

$$
\begin{aligned}
& \Phi(r)=\frac{2 q}{\epsilon_{2} r} \mathcal{I}_{a}(\kappa r), \\
& E_{z,+}(r)=-\frac{2 q}{\epsilon_{2} r^{2}} \mathcal{I}_{b}(\kappa r), \\
& \mathbf{E}_{\|}(r)=-\frac{d \Phi}{d r} \mathbf{e}_{r}=\frac{2 q}{\epsilon_{2} r^{2}} \mathcal{I}_{c}(\kappa r) \mathbf{e}_{r},
\end{aligned}
$$

where $\mathbf{e}_{r}$ is the unit radial vector pointing away from the center of the colloid, and the auxiliary functions $\mathcal{I}_{n}(k)$ are given by integrals over a Bessel function,

$$
\begin{aligned}
& \mathcal{I}_{a}(k):=\int_{0}^{\infty} d x J_{0}(x) \frac{x}{\left(\epsilon_{1} / \epsilon_{2}\right) x+\sqrt{x^{2}+k^{2}}}, \\
& \mathcal{I}_{b}(k):=\frac{\epsilon_{2}}{\epsilon_{1}} \int_{0}^{\infty} d x J_{0}(x) \frac{x \sqrt{x^{2}+k^{2}}}{\left(\epsilon_{1} / \epsilon_{2}\right) x+\sqrt{x^{2}+k^{2}}},
\end{aligned}
$$

These integrals have been computed numerically; the analytic expressions for their asymptotic behaviors are derived in Appendix D. Thus, taking $\epsilon_{2} / \epsilon_{1} \gg 1$ (the ratio $\epsilon_{2} / \epsilon_{1}$ is approximately 81 for water in contact with air) in the intermediate asymptotic regime $1 \ll \kappa r \ll\left(\epsilon_{2} / \epsilon_{1}\right)^{2}$, one obtains the following expressions for the potential and the field components [see Eqs. (42a)-(42c)]:

$$
\begin{aligned}
& \Phi(r) \approx \frac{2 q}{\epsilon_{2} r}\left[\frac{\epsilon_{1}}{\epsilon_{2}} \frac{1}{(\kappa r)^{2}}+\mathrm{e}^{-\kappa r}\right], \\
& E_{z,+}(r) \approx \frac{2 q}{\epsilon_{2} r^{2}}\left[-\frac{1}{\kappa r}+\sqrt{\frac{\pi}{2}} \frac{\epsilon_{1}}{\epsilon_{2}}(\kappa r)^{3 / 2} \mathrm{e}^{-\kappa r}\right], \\
& \mathbf{E}_{\|}(r) \approx \frac{2 q}{\epsilon_{2} r^{2}}\left[3 \frac{\epsilon_{1}}{\epsilon_{2}} \frac{1}{(\kappa r)^{2}}+\kappa r \mathrm{e}^{-\kappa r}\right] \mathbf{e}_{r} .
\end{aligned}
$$

Asymptotically, $\Phi(r)$ decays as $1 / r^{3}$ and the electrostatic interaction energy $q \Phi(d)$ of a second charge $q$ at a distance $d$ from the first one decays likewise as $1 / d^{3}$. This is the celebrated dipole repulsion between charged colloidal particles first conjectured in Ref. 1. However, due to the large value of the ratio $\epsilon_{2} / \epsilon_{1}$, closer to the charge there is a crossover to a screened Coulomb potential $\Phi(r) \propto \exp (-\kappa r) / r$. One can introduce a crossover length $r_{\text {cross }}$ defined from the asymptotic behavior given by Eqs. (44a)-(44c) as

$$
\frac{\epsilon_{1}}{\epsilon_{2}} \frac{1}{\left(\kappa r_{\text {cross }}\right)^{2}}=\mathrm{e}^{-\kappa r_{\text {cross }}}
$$

This equation has solutions only if $\epsilon_{1} / \epsilon_{2}<(2 / \mathrm{e})^{2} \approx 0.54$, in which case the relevant solution $\kappa r_{\text {cross }}$ is larger than 2 and depends only weakly, i.e., logarithmically on the precise value of the ratio $\epsilon_{1} / \epsilon_{2}$. For $\epsilon_{2} / \epsilon_{1}=81$, this gives $\kappa r_{\text {cross }}$ $\approx 8.7$. The parallel component $\mathbf{E}_{\|}$exhibits the same crossover, but the normal component $E_{z,+}$ always decays algebraically and is much larger than the parallel component. Nevertheless, the contribution of $E_{z,+}$ to the pressure field $\Pi(r)$ is reduced by a factor $\epsilon_{1} / \epsilon_{2}$ [see Eq. (38)], so that $\Pi(r)$ will also exhibit the crossover at a distance $r \approx r_{\text {cross }}$. From Eqs. (38) and (39), one finds

$$
\begin{aligned}
\Pi(r)= & \gamma r_{0} \kappa^{2} \frac{\varepsilon_{F}}{(\kappa r)^{4} \mathcal{P}\left(\kappa r_{0}\right)}\left[\frac{\epsilon_{1}}{\epsilon_{2}}\left(1-\frac{\epsilon_{1}}{\epsilon_{2}}\right) \mathcal{I}_{b}^{2}(\kappa r)\right. \\
& \left.+\left(1-\frac{\epsilon_{1}}{\epsilon_{2}}\right) \mathcal{I}_{c}^{2}(\kappa r)+(\kappa r)^{2} \mathcal{I}_{a}^{2}(\kappa r)\right],
\end{aligned}
$$

where [see Eq. (1)]

$$
\varepsilon_{F}=\varepsilon_{\Pi}=\frac{q^{2} \kappa^{2}}{2 \pi \epsilon_{2} \gamma r_{0}} \mathcal{P}\left(\kappa r_{0}\right),
$$

with the dimensionless function 


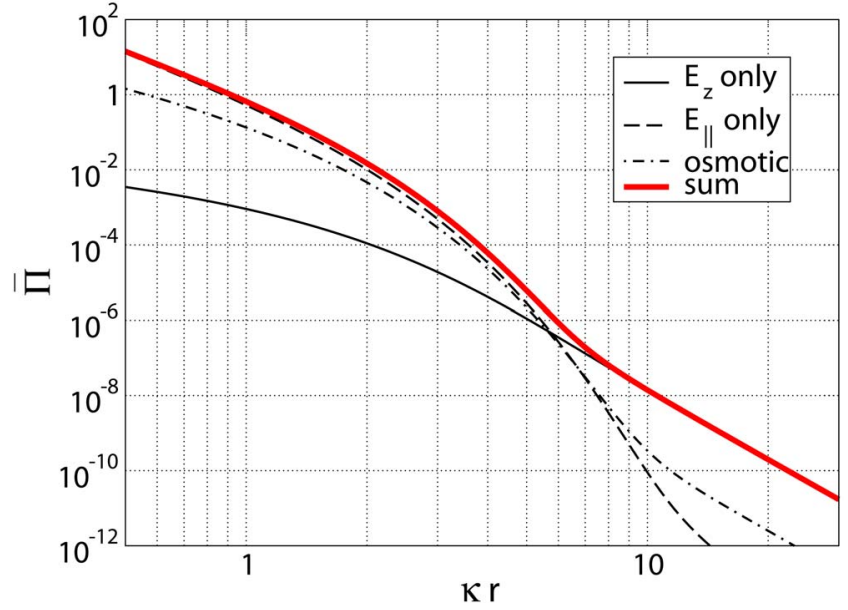

FIG. 3. (Color online) The dimensionless stress $\bar{\Pi}:=\Pi /\left[\gamma r_{0} \kappa^{2} \varepsilon_{F} / \mathcal{P}\left(\kappa r_{0}\right)\right]$ due to a single point charge at the interface for $\varepsilon_{2} / \varepsilon_{1}=81$, as given by Eq (46) (thick solid line). The thin lines represent each of the three additive contributions in Eq. (46): due to $E_{z}$, only given by $\mathcal{I}_{b}$; due to $E_{\|}$, only given by $\mathcal{I}_{c}$; and due to the osmotic pressure, only given by $\mathcal{I}_{a}$. As expected, one observes a crossover in $\bar{\Pi}$ at a distance $r$ comparable with $r_{\text {cross }}$ defined by Eq. (45).

$$
\begin{aligned}
\mathcal{P}\left(\kappa r_{0}\right):= & \int_{\kappa r_{0}}^{\infty} d x \frac{1}{x^{3}}\left[\frac{\epsilon_{1}}{\epsilon_{2}}\left(1-\frac{\epsilon_{1}}{\epsilon_{2}}\right) \mathcal{I}_{b}^{2}(x)\right. \\
& \left.+\left(1-\frac{\epsilon_{1}}{\epsilon_{2}}\right) \mathcal{I}_{c}^{2}(x)+x^{2} \mathcal{I}_{a}^{2}(x)\right] \\
\approx & {\left[\frac{1}{\kappa r_{0}}+\frac{1}{2\left(\kappa r_{0}\right)^{2}}\right] \mathrm{e}^{-2 \kappa r_{0}}, }
\end{aligned}
$$

where the last line is the leading (zeroth order) contribution in an expansion in terms of the small parameter $\epsilon_{1} / \epsilon_{2}$. Equation (47) allows one to express the parameter $q$, the value of which is often uncertain, in terms of the more convenient parameter $\varepsilon_{F}$. Figure 3 shows how $\Pi(r)$ attains the dipole limiting behavior $\propto 1 / r^{6}$ due to the electrostatic pressure $\propto E_{z,+}^{2}$ beyond the crossover distance; at shorter distances, $\Pi(r)$ is dominated by the term $\propto \mathbf{E}_{\|}^{2}$ and, to a lesser extent, by the osmotic pressure. The figure indicates also that the main contribution to the total force $\varepsilon_{\Pi}$ stems from the regions close to the particle, as evidenced also by the formal divergence of $\mathcal{P}\left(\kappa r_{0} \rightarrow 0\right)$ [see Eq. (48)]. Thus, the precise value of $\varepsilon_{\Pi}$ will be affected by the fact that our solution of the electrostatic problem within the monopolar approximation is expected to be reliable, in principle, only sufficiently far from the particle.

We consider now two identical point charges at the interface separated by a distance $d$. Within the Debye-Hückel and point-charge approximations, the solution of this electrostatic problem is given by the superposition of the singlecolloid fields. In this case, the field $\Pi_{m}(\mathbf{r})$ in Eq. (6a) reads as follows [as in Sec. II A, the subscript $\alpha(=1,2)$ denotes that the corresponding field is evaluated with respect to particle $\alpha$; see Fig. 2 for the notation]:

$$
\begin{aligned}
\Pi_{m}(r)= & \frac{\epsilon_{2}-\epsilon_{1}}{8 \pi}\left[\frac{\epsilon_{1}}{\epsilon_{2}}\left(E_{z,+}\right)_{1}\left(E_{z,+}\right)_{2}+\left(\mathbf{E}_{\|}\right)_{1} \cdot\left(\mathbf{E}_{\|}\right)_{2}\right] \\
& +\frac{\epsilon_{2}}{8 \pi} \kappa^{2} \Phi_{1} \Phi_{2} \\
= & \frac{q^{2} \kappa^{4}}{2 \pi \epsilon_{2}} \frac{1}{\kappa^{4}\left(\left|\mathbf{r}-\mathbf{r}_{1}\right|\left|\mathbf{r}-\mathbf{r}_{2}\right|\right)^{2}}\left[\frac{\epsilon_{1}}{\epsilon_{2}}\left(1-\frac{\epsilon_{1}}{\epsilon_{2}}\right) \mathcal{I}_{b}(\kappa \mid \mathbf{r}\right. \\
& \left.-\mathbf{r}_{1} \mid\right) \mathcal{I}_{b}\left(\kappa\left|\mathbf{r}-\mathbf{r}_{2}\right|\right)+\left(1-\frac{\epsilon_{1}}{\epsilon_{2}}\right) \mathcal{I}_{c}\left(\kappa\left|\mathbf{r}-\mathbf{r}_{1}\right|\right) \mathcal{I}_{c}(\kappa \mid \mathbf{r} \\
& \left.-\mathbf{r}_{2} \mid\right) \frac{\mathbf{r}-\mathbf{r}_{1}}{\left|\mathbf{r}-\mathbf{r}_{1}\right|} \cdot \frac{\mathbf{r}-\mathbf{r}_{2}}{\left|\mathbf{r}-\mathbf{r}_{2}\right|}+\kappa^{2}\left|\mathbf{r}-\mathbf{r}_{1}\right|\left|\mathbf{r}-\mathbf{r}_{2}\right| \mathcal{I}_{a}(\kappa \mid \mathbf{r} \\
& \left.\left.-\mathbf{r}_{1} \mid\right) \mathcal{I}_{a}\left(\kappa\left|\mathbf{r}-\mathbf{r}_{2}\right|\right)\right]
\end{aligned}
$$

Therefore, one has [see Eqs. (15b) and (47)]

$$
\varepsilon_{\Pi_{m}}=\frac{\mathcal{P}_{m}\left(\kappa r_{0}, \kappa d\right)}{\mathcal{P}\left(\kappa r_{0}\right)} \varepsilon_{F},
$$

where

$$
\begin{aligned}
\mathcal{P}_{m}\left(\kappa r_{0}, \kappa d\right):= & \frac{1}{2 \pi} \int_{\hat{S}_{\text {men }}} d^{2} \mathbf{x} \frac{1}{\left(x_{1} x_{2}\right)^{2}} \\
& \times\left[\frac{\epsilon_{1}}{\epsilon_{2}}\left(1-\frac{\epsilon_{1}}{\epsilon_{2}}\right) \mathcal{I}_{b}\left(x_{1}\right) \mathcal{I}_{b}\left(x_{2}\right)\right. \\
& +\left(1-\frac{\epsilon_{1}}{\epsilon_{2}}\right) \mathcal{I}_{c}\left(x_{1}\right) \mathcal{I}_{c}\left(x_{2}\right) \frac{\mathbf{x}_{1} \cdot \mathbf{x}_{2}}{x_{1} x_{2}} \\
& \left.+x_{1} x_{2} \mathcal{I}_{a}\left(x_{1}\right) \mathcal{I}_{a}\left(x_{2}\right)\right]
\end{aligned}
$$

in terms of $\mathbf{x}:=\kappa \mathbf{r}$ and $\mathbf{x}_{\alpha}:=\kappa\left(\mathbf{r}-\mathbf{r}_{\alpha}\right)$. Dependences on $r_{0}$ and $d$ enter through the specification of the integration domain $\hat{S}_{\text {men. }}$. Using this expression and Eqs. (43a)-(43c), we have integrated numerically Eqs. (8a)-(8c) using bipolar coordinates in a domain corresponding to a size $L \approx 100 / \kappa$. Taking this numerical solution and the exact expression in Eq. (A1), we have subsequently computed numerically the effective interaction energy as $V_{\text {men }}(d) \approx{ }^{b} V_{\text {corr }}(d)$ by using Eq. (10b). As in the case studied in Sec. III A [see the text after Eq. (34)], the last term in Eq. (10b) turns out to be larger in magnitude than the remaining terms, but they are approximately proportional to each other, so that for the purpose of understanding the numerical results, we can consider the following proportionality [upon the application of Eq. (18)]:

$$
\begin{aligned}
& V_{\text {men }}(d) \propto-2 \pi \gamma r_{0}^{2} \varepsilon_{m}\left(2 \varepsilon_{F}+\varepsilon_{m}\right) \sim-4 \pi \gamma r_{0}^{2} \varepsilon_{F} \varepsilon_{\Pi_{m}} \\
& =-4 \pi \gamma r_{0}^{2} \varepsilon_{F}^{2} \frac{\mathcal{P}_{m}\left(\kappa r_{0}, \kappa d\right)}{\mathcal{P}\left(\kappa r_{0}\right)} .
\end{aligned}
$$

Figure 4 shows indeed that for two choices of $\kappa r_{0}$ this approximation is reasonable at large $d$. Numerically, we find that the term $\propto \varepsilon_{F} \varepsilon_{\Pi_{m}}$ contributes $\sim 70 \%$ of the total meniscus-induced potential $V_{\text {men }}$. Asymptotically, for large $d$ it exhibits the predicted $1 / d^{3}$ behavior [Eq. (27) with $n=6$ ]. More precisely, the asymptotic behavior of $V_{\text {men }}$ can be ob- 


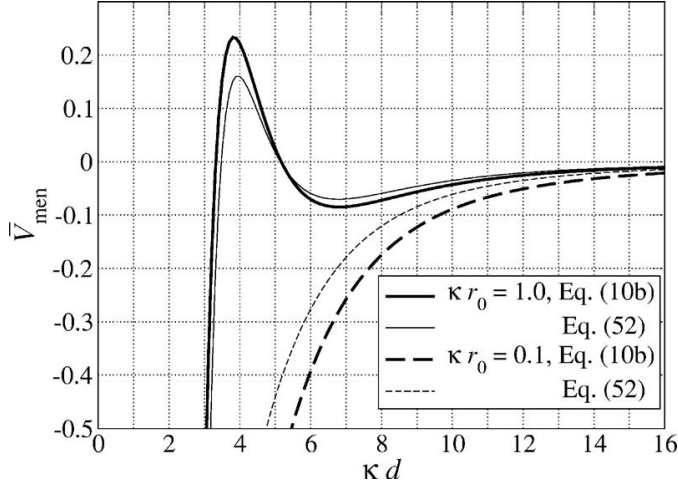

FIG. 4. The dimensionless capillary-induced potential energy $\bar{V}_{\text {men }}$ $:=10^{2} V_{\text {men }} /\left[\gamma r_{0}^{2} \varepsilon_{F}^{2} / \mathcal{P}\left(\kappa r_{0}\right)\right]$ for two different values of $\kappa r_{0}$ and the choice $\epsilon_{2} / \epsilon_{1}=81$. Thick lines correspond to the capillary-induced potential given by Eq. (10b) [see Eq. (27)], whereas thin lines show the approximation $\propto \varepsilon_{F} \varepsilon_{\Pi_{m}}$ given by Eq. (52). If $\kappa r_{0}$ is large enough, a minimum appears.

tained as an expansion in terms of $1 / d$ by utilizing the twopeak structure of $\Pi_{m}$ (as for the general discussion in Sec. II B): One assumes that for $d \rightarrow \infty$ the main contribution to the integral in Eq. (51) stems from the regions near the colloidal particles. The leading asymptotic behavior of $\mathcal{P}_{m}$ is given simply by the lowest-order term of a Taylor expansion about $x_{1} \approx \kappa r_{0}, x_{2} \approx \kappa d$ and $x_{1} \approx \kappa d, x_{2} \approx \kappa r_{0}$ in the integral in Eq. (51) with the asymptotic decay for the $\mathcal{I}$ functions obtained in Appendix D, ${ }^{39}$

$$
\mathcal{P}_{m}\left(\kappa r_{0}, \kappa d\right) \approx \frac{\epsilon_{1}}{\epsilon_{2}} \frac{p_{m}\left(\kappa r_{0}\right)}{(\kappa d)^{3}},
$$

where

$$
\begin{aligned}
p_{m}\left(\kappa r_{0}\right) & :=2 \int_{\kappa r_{0}}^{\infty} d x\left[\left(1-\frac{\epsilon_{1}}{\epsilon_{2}}\right) \frac{\mathcal{I}_{b}(x)}{x}+\mathcal{I}_{a}(x)\right] \\
& \approx 2\left[\kappa r_{0} I_{0}\left(\frac{\kappa r_{0}}{2}\right) K_{1}\left(\frac{\kappa r_{0}}{2}\right)-1+\mathrm{e}^{-\kappa r_{0}}\right],
\end{aligned}
$$

and the second line is the leading (zeroth order) contribution in an expansion in terms of the small parameter $\epsilon_{1} / \epsilon_{2}$, obtained from applying Eqs. (D2) and (D13). According to this expression, $p_{m}\left(\kappa r_{0} \rightarrow 0\right)=4$ and $p\left(\kappa r_{0}\right)$ decreases monotonously with an asymptotic decay $p\left(\kappa r_{0} \rightarrow \infty\right) \sim 2 /\left(\kappa r_{0}\right)$. This result for $\mathcal{P}_{m}\left(\kappa r_{0}, \kappa d\right)$ means that the asymptotic $1 / d^{3}$ decay is determined by the normal component $E_{z}(d)$ of the field and by the potential $\Phi(d)$ stemming from the osmotic pressure. There is no contribution from $\mathbf{E}_{\|}$to the leading order in $1 / d$ due to the geometrical factor $\mathbf{x}_{1} \cdot \mathbf{x}_{2}$ appearing in Eq. (51). Finally, from Fig. 4 we infer that if $\kappa r_{0}$ is large enough, the asymptotic decay breaks down as a reliable approximation and a minimum appears in $V_{\text {men }}(d)$ at a separation $d$ which is a few times $\kappa^{-1}$.

The energy due to the direct repulsion of the two colloids is given by Eq. (42a),

$$
V_{\text {rep }}(d)=q \Phi(d)=4 \pi \gamma r_{0}^{2} \varepsilon_{F} \frac{\mathcal{I}_{a}(\kappa d)}{(\kappa d)\left(\kappa r_{0}\right) \mathcal{P}\left(\kappa r_{0}\right)},
$$

and the total energy is $V_{\text {tot }}(d)=V_{\text {rep }}(d)+V_{\text {men }}(d)$. With approximation (52), one has

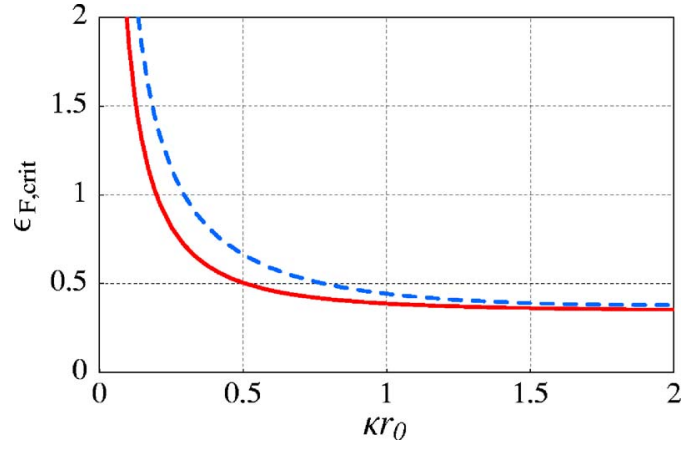

FIG. 5. (Color online) The solid line gives the critical value $\varepsilon_{F \text {,crit }}$ as a function of $\kappa r_{0}$ specified by the fit to the numerical results [Eq. (59)]. The dashed line corresponds to the approximate expression provided by Eq. (58).

$$
V_{\mathrm{tot}}(d) \approx \frac{4 \pi \gamma r_{0}^{2} \varepsilon_{F}}{\kappa r_{0} \mathcal{P}\left(\kappa r_{0}\right)}\left[\frac{\mathcal{I}_{a}(\kappa d)}{\kappa d}-\varepsilon_{F} \kappa r_{0} \mathcal{P}_{m}\left(\kappa r_{0}, \kappa d\right)\right]
$$

Asymptotically, for $\kappa d \gg 1$ this expression reduces to [see Eqs. (53) and (D3)]

$$
\begin{aligned}
V_{\mathrm{tot}}(d) \sim & 4 \pi \gamma r_{0}^{2} \varepsilon_{F} \frac{\epsilon_{1} / \epsilon_{2}}{\left(\kappa r_{0}\right)^{4} \mathcal{P}\left(\kappa r_{0}\right)}\left(\frac{r_{0}}{d}\right)^{3} \\
& \times\left[1+\frac{\epsilon_{2}}{\epsilon_{1}}(\kappa d)^{2} \mathrm{e}^{-\kappa d}-\kappa r_{0} p_{m}\left(\kappa r_{0}\right) \varepsilon_{F}\right],
\end{aligned}
$$

to be compared with the potential obtained in the previous subsection [Eq. (35)]. As in that case, the capillary-induced potential $V_{\text {men }}$ is reduced by a factor $\varepsilon_{F} \ll 1$ with respect to $V_{\text {rep }}$, and the total potential can be asymptotically attractive only if $\varepsilon_{F}$ is above a critical value,

$$
\varepsilon_{F, \text { crit }}=\frac{1}{\kappa r_{0} p_{m}\left(\kappa r_{0}\right)},
$$

which to the leading order is independent of the small ratio $\epsilon_{1} / \epsilon_{2}$ and turns out to be bounded as $\varepsilon_{F, \text { crit }}>0.38$ (see Fig. 5). Moreover, we note that even if $\varepsilon_{F} \sim 1$ the term in brackets in Eq. (57) changes sign at a separation $d$ comparable with the crossover length $r_{\text {cross }}$ [see Eq. (45)]. This is a consequence of the crossover in $V_{\text {rep }}(d) \propto \Phi(d)$ and suggests that although the potential is asymptotically attractive, it reaches a minimum at a distance $d \approx r_{\text {cross }}$ and turns repulsive for closer separations. This effect can only be enhanced by the deviation from the asymptotic $1 / d^{3}$ decay in $V_{\text {men }}(d)$ observed in Fig. 4 for $\kappa r_{0} \sim 1$.

These conclusions based on approximation (52) are supported by the corresponding full numerical calculations. More precisely, from a fit to these numerical results in the range $0.1 \leqslant \kappa r_{0} \leqslant 2.0$ for the ratio $\epsilon_{2} / \epsilon_{1}=81$, we find that the critical value is given approximately by

$$
\varepsilon_{F, \text { crit }}\left(\kappa r_{0}\right) \approx 0.34 \sqrt{1+\frac{0.30}{\left(\kappa r_{0}\right)^{2}}} .
$$

Figure 6 shows a plot of $V_{\text {tot }}(d)$ for a typical value $\varepsilon_{F}=0.6$, exhibiting a shallow minimum if it is asymptotically attractive. In summary, the capillary-induced attraction can dominate asymptotically only if $\kappa r_{0}$ is sufficiently large so that $\varepsilon_{F}>\varepsilon_{F \text {,crit }}$, in which case one necessarily has $\varepsilon_{F} \gtrsim 1$. 


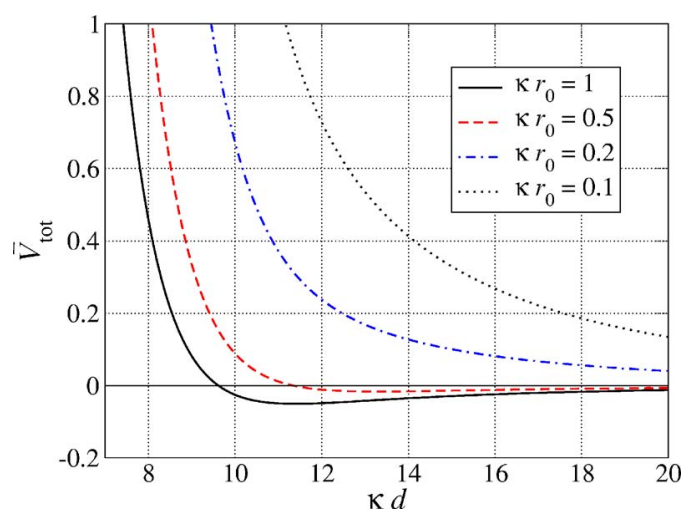

FIG. 6. (Color online) The dimensionless total potential energy $\bar{V}_{\text {to }}$ $:=10^{3} V_{\text {tot }} /\left[\gamma r_{0}^{2} \varepsilon_{F} / \mathcal{P}\left(\kappa r_{0}\right)\right]$ for different values of $\kappa r_{0}$ and the choices $\epsilon_{2} / \epsilon_{1}$ $=81$ and $\varepsilon_{F}=0.6$. Note that the total energy is about ten times smaller than $V_{\text {men }}$ (the normalization of $\bar{V}_{\text {tot }}$ here differs from that of $\bar{V}_{\text {men }}$ in Fig. 4 by a factor of $10 / \varepsilon_{F}$ ) and exhibits a shallow minimum provided that $\kappa r_{0}$ is not too small.

\section{SUMMARY AND DISCUSSION}

We have carried out a thorough analysis of the effective interaction potential $V_{\text {men }}(d)$ between two colloidal particles floating a distance $d$ apart from each other at a fluid interface due to the deformation of the interface caused by the particles. The main result is summarized in Eq. (27) for the asymptotic behavior of $V_{\text {men }}(d)$ as $d \rightarrow \infty$. One finds two qualitatively different cases depending on the (dimensionless) force $\varepsilon_{F}$ acting on the particle and the one, $\varepsilon_{\Pi}$, acting on the interface [see Eqs. (1) and (14)]: (a) If the system is not mechanically isolated $\left(\varepsilon_{F}-\varepsilon_{\Pi} \neq 0\right)$, the superposition approximation is valid and the asymptotic dependence $V_{\text {men }}(d$ $\rightarrow \infty$ ) is universal in the sense of being independent of the detailed distribution of the forces which deform the interface. The physical reason for this is that each particle, together with its surrounding interface, can be considered as an effective particle. (b) In the opposite case of mechanical isolation $\left(\varepsilon_{F}-\varepsilon_{\Pi}=0\right)$, the corrections to the superposition ansatz are dominant and the asymptotic decay of $V_{\text {men }}(d)$ depends on the asymptotic properties of the pressure field acting on the interface. We identified two sources of violation of the superposition approximation: The boundary conditions of the deformation at the particle-interface contact line are violated, and the pressure field acting on the interface does not satisfy a superposition principle.

These results hold under the rather general assumptions that (i) the interfacial deformation is small $\left(\varepsilon_{F}, \varepsilon_{\Pi} \ll 1\right)$, (ii) the pressure field $\hat{\Pi}=\Pi_{1}+\Pi_{2}+2 \Pi_{m}$ exerted by the particles on the interface satisfies the scaling relations in Eqs. (12) and (13), and (iii) the external force acting on the particles is additive [Eq. (14)]. Assumptions (i) and (iii) are quite general, and there is no evidence that they are not fulfilled experimentally. Assumption (ii) can be viewed as a consequence of the condition that the pressure $\hat{\Pi}$ is derivable from the stress tensor of a Lagrangian quadratic in an underlying field, as it is typically the case for physical systems: Besides stresses induced by electric fields as addressed here, there can be, e.g., elastic stresses which arise if one of the fluids is in a nematic phase. $^{40}$ (This latter case also provides an ex- ample in which the asymptotic decay [Eq. (27)] is actually modified by geometrical constraints which complement the scaling relation [Eq. (12)].)

As an application for the case of mechanical isolation, we have considered the paradigmatic system of electrically charged colloids (see Figs. 1 and 2), which is also of direct experimental importance. The pressure deforming the interface is due to the electrostatic field emanating from the particles and gives rise to an effective attractive interaction $V_{\text {men }}(d \rightarrow \infty) \sim 1 / d^{3}$. Since the direct electric repulsion between the colloids also decays $V_{\text {rep }} \sim 1 / d^{3}$, this asymptotic analysis is insufficient to determine whether the total potential $V_{\text {men }}+V_{\text {rep }}$ describes an asymptotically attractive force, as seemingly observed experimentally. To this end, as a model for the experiments, it is necessary to consider in detail the challenging electrostatic problem posed by two charged spheres partially immersed in an electrolyte. Within this approach, we have studied the electrostatic problem in two simplifying limiting regimes: (A) water as one of the fluid phases is a perfect conductor, and (B) the colloidal particles are replaced by point charges (monopolar approximation). Within both approximations, we concluded that $V_{\text {men }} \propto \varepsilon_{F}^{2}$, while $V_{\text {rep }} \propto \varepsilon_{F}$, so that the direct repulsion dominates asymptotically; the capillary-induced attraction is predicted to dominate only if $\varepsilon_{F}$ is larger than a threshold value $\varepsilon_{F \text {,crit }}$ $\approx 1$ [see Eqs. (36) and (59)]; i.e., the deformation of the interface has to be large, which is outside the range of validity of the present analysis based on small deformations $\left(\varepsilon_{F}\right.$ $\ll 1)$.

Assumption (A) is a simple model of the experimentally relevant case of polystyrene or glass colloids floating at the interface between salty water and air or oil such that the Debye length $\kappa^{-1}$ of water is much smaller than the radius of the particle-interface contact line $r_{0}$. Only the residual charges at the interface between the colloid and the air or oil phase determine $\varepsilon_{F}$. The charge density $\sigma$ at this interface is rather small compared with the nominal surface density of dissociable groups; we note that in the case of polystyrene colloids the precise microscopic origin of this charge density is still unknown. By dimensional analysis, $\varepsilon_{F}$ $=\left(\sigma^{2} r_{0} / \epsilon_{1} \gamma\right) G\left(\epsilon_{C} / \epsilon_{1}, \theta\right)$, where $G$ is a dimensionless function depending on the contact angle $\theta$ and the ratio of dielectric constants of the colloidal particle and the insulating fluid, $\epsilon_{C}$ and $\epsilon_{1}$, respectively. The electrostatic solution ${ }^{37}$ yields $G(1, \theta) \approx 3 / \sin ^{3} \theta$ for hydrophobic colloids. In Ref. 41 the single-colloid meniscus deformation was measured around rather large glass spheres $\left(r_{0} \approx 200 \mu \mathrm{m}\right)$ at a water-oil interface $\left(\gamma \approx 0.05 \mathrm{~N} / \mathrm{m}, \epsilon_{1} \approx 2\right)$, which are slightly hydrophobic $\left(\theta \approx 120^{\circ}\right)$. Using Eq. (A1), a value $\varepsilon_{F} \sim 0.4$ was inferred from the measurements, corresponding to a charge density of $\sigma \sim 70 \mu \mathrm{C} / \mathrm{m}^{2}$ (or $5 \times 10^{-4} \mathrm{e} / \mathrm{nm}^{2}$ ) according to the simple formula given above. This value is close to $\varepsilon_{F, \text { crit }}$ given in Eq. (36), and thus one could expect a strongly reduced repulsion or even a net attraction between pairs of these glass spheres. A corresponding extension of this single-sphere experiment would be highly desirable.

For truly nanoscopic colloids $\left(r_{0} \lesssim 1 \mu \mathrm{m}\right)$ with this same charge density on the air or the oil side, the formula above predicts $\varepsilon_{F}=O\left(10^{-3}\right)$; therefore, the electrostatic repulsion 
would always dominate the capillary attraction. This is consistent with the results in Ref. 34 obtained for polystyrene spheres $\left(r_{0} \approx 1 \mu \mathrm{m}\right)$ at the oil/water interface. For highly salty water, the charge density on the colloid/oil interface is estimated experimentally to be $\sigma \sim 20 \mu \mathrm{C} / \mathrm{m}^{2}$, and force measurements between two spheres confirmed the repulsive dipole-dipole interaction with no sign of capillary attraction.

Assumption (B) amounts to modeling a system in which the Debye length of water is comparable or larger than the radius $r_{0}$. We have studied in detail the case that the dielectric constant of one fluid phase (e.g., water) is much larger than the dielectric constant of the other fluid phase (e.g., air). The capillary-induced potential $V_{\text {men }}$ is asymptotically attractive (see Fig. 4), but the total potential $V_{\text {men }}+V_{\text {rep }}$ can be asymptotically attractive for values $\varepsilon_{F} \sim 1$ only if $\kappa r_{0} \geq 1$ (see Fig. 5). However, unlike the model corresponding to assumption (A), even in such a case $V_{\text {men }}+V_{\text {rep }}$ becomes repulsive at small separations (Fig. 6). This effect can be traced back to a crossover in the interfacial stress $\Pi(r)$ from the asymptotic algebraic decay [as in model (A)] to an exponential decay at closer distances $r \lesssim 7 / \kappa$ from the particle (see Fig. 3).

In contrast to the experiments carried out with nano- and microcolloids at interfaces with salty water, some experiments $^{9,10,12-14}$ have been performed with microcolloids at interfaces of ultrapure water such that $\kappa^{-1} \sim r_{0} \sim 1 \mu \mathrm{m}$. These experiments could have explored phenomena beyond the small-deformation regime. Equation (47) provides a relationship between $\varepsilon_{F}$ and the relevant parameters of the experimental system; regrettably, the value of the total charge $q$ is usually uncertain. In terms of the surface charge density $\sigma$, one has $q=2 \pi \sigma\left(r_{0} \sin \theta\right)^{2}(1+\cos \theta)$ because for ultrapure water the electrostatic field is dominated by the unscreened charge of the particle on the water side. For typical values $\epsilon_{2}=81, \gamma=0.05 \mathrm{~N} / \mathrm{m}, \kappa=1 \mu \mathrm{m}^{-1}$, and $\theta=\pi / 2$ (so that $r_{0}$ $=$ radius of the particle), Eq. (47) gives $\varepsilon_{F} \approx 371 \sigma^{2} r_{0}^{3} \mathcal{P}\left(r_{0}\right)$, with $r_{0}$ in micrometers and $\sigma$ in units of $e / \mathrm{nm}^{2}$. The values for $\sigma$ quoted in the literature range from $0.07 \mathrm{e} / \mathrm{nm}^{2}$ (Ref. 7) to $0.53 \mathrm{e} / \mathrm{nm}^{2}$ (Ref. 8). Accordingly, Fig. 7 shows that it seems possible to have capillary attraction (i.e., $\varepsilon_{F}>\varepsilon_{F, \text { crit }}$ $\approx 1$ ) for typical values of the particle radius in the micrometer range. However, we emphasize that, unlike the conclusion concerning the asymptotic decay of $V_{\text {men }}$, the expressions relating the value of $\varepsilon_{F}$ and $\varepsilon_{F \text {,crit }}$ with the parameters of the system involve the behavior of the electric field near the particle [see Eqs. (48) and (53)]. Therefore, they are expected to be affected by corrections to the monopolar approximation and the Debye-Hückel approximation. ${ }^{42}$ Thus, further theoretical work is required to understand these experiments properly and to arrive at reliable predictions.

\section{ACKNOWLEDGMENTS}

One of the authors (A.D.) acknowledges financial support from the Junta de Andalucía (Spain). Another author (M.O.) acknowledges financial support from the German Science Foundation (DFG) through the Collaborative Research Centre "Colloids in External Fields" (SFB-TR6).

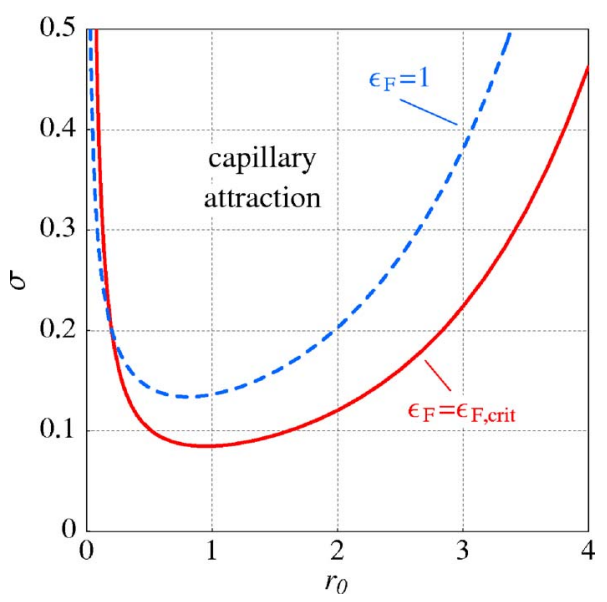

FIG. 7. (Color online) Parameter space spanned by the surface charge density $\sigma$ (in units of $e / \mathrm{nm}^{2}$ ) and the contact line radius $r_{0}$ (in $\mu \mathrm{m}$ ) (see text for the fixed values of the other parameters). The solid line corresponds to the loci $\varepsilon_{F}=\varepsilon_{F \text {,crit }}$, so that capillary attraction is predicted to occur in systems the parameters of which fall into the region above this curve. As a reference curve, the dashed line corresponds to the loci $\varepsilon_{F}=1$.

\section{APPENDIX A: INTERFACE DEFORMATION FIELDS}

In this appendix, we derive the single-colloid deformation $u(r)$ and the correction $u_{m}(\mathbf{r})$ to the superposition approximation for two colloids.

The deformation field $u(r)$ in the single-colloid configuration is readily obtained as the rotationally symmetric solution of Eqs. (5a)-(5c),

$$
\begin{aligned}
u(r) & =r_{0}\left(\varepsilon_{\Pi}-\varepsilon_{F}\right) \ln \frac{L}{r}-\frac{1}{\gamma} \int_{r}^{L} d s s \Pi(s) \ln \frac{s}{r} \\
& \sim\left\{\begin{array}{ll}
r^{2-n} & \text { if } \varepsilon_{F}-\varepsilon_{\Pi}=0 \\
\ln r & \text { if } \varepsilon_{F}-\varepsilon_{\Pi} \neq 0
\end{array} \quad\left(r_{0} \ll r \ll L \text { and } n>2\right) .\right.
\end{aligned}
$$

The limit $L \rightarrow \infty$ is well defined if $\varepsilon_{F}-\varepsilon_{\Pi}=0$; otherwise, the presence of the boundary condition [Eq. $(5 \mathrm{c})]$ is required to regularize the possible logarithmic divergence. ${ }^{22}$ The physi-

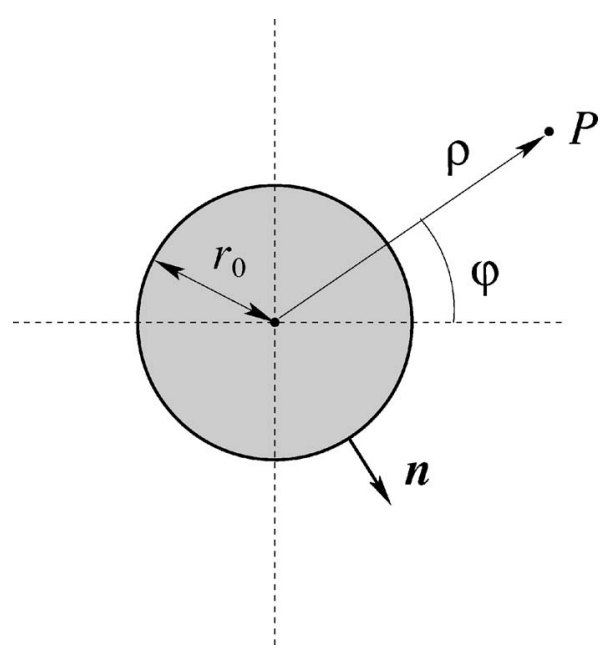

FIG. 8. The position of a point $P$ near a colloid is parametrized by the polar coordinates $(\rho, \varphi)$. The unit vector normal to the (circular) contact line is $\mathbf{n}=\mathbf{e}_{\rho}$. 
cal interpretation of this regularization is a force acting on the boundary $C_{L}$ of the interface, which compensates the net force $2 \pi \gamma r_{0}\left(\varepsilon_{F}-\varepsilon_{\Pi}\right)$ localized around the particle. Since these two forces act at well separated locations, there is an intermediate range of lengths where there is approximately no force acting on the interface, so that the corresponding deformation varies logarithmically. (The electrostatic analogy developed in Ref. 27 provides a transparent visualization of this explanation.)

The correction $u_{m}(\mathbf{r})$ to the superposition approximation cannot be computed analytically in an easy manner, but we shall derive several asymptotic properties of the solution as the interparticle separation $d$ becomes large. In order to follow the arguments, the reader will find the electrostatic analogy useful, which relies on the formal analogy of Eqs. (8a)-(8c) with the equations for the two-dimensional electrostatic potential: $u_{m}$ plays the role of the potential and $2 \Pi_{m}$ the role of the charge density. (This analogy is worked out in detail in Ref. 27.)

First, we approximately compute the function $u_{m}(\mathbf{r})$ near the colloids, i.e., at distances $r_{0} \leqslant \rho:=\left|\mathbf{r}-\mathbf{r}_{\alpha}\right| \ll d$ from the particle (see Fig. 8). Concerning the boundary condition [Eq. (8b)] at $\rho=r_{0}$, we note that

$$
\begin{aligned}
-\mathbf{n}_{\alpha} & \cdot \nabla u_{\beta}+\frac{u_{\beta}-\left\langle u_{\beta}\right\rangle}{r_{0}} \\
& \approx \varepsilon_{12}-\frac{r_{0}}{2}\left[\mathbf{n n}: \nabla \nabla u(d)-\frac{1}{2} \nabla^{2} u(d)\right] \\
& \approx \varepsilon_{12}-\frac{r_{0}}{4}\left[u^{\prime \prime}(d)-\frac{u^{\prime}(d)}{d}\right] \cos 2 \varphi,
\end{aligned}
$$

which follows from expanding the single-particle solution [Eq. (A1)] around $r=d$, where one finds [see Eq. (A1)]

$$
u^{\prime \prime}(d)-\frac{u^{\prime}(d)}{d} \sim \begin{cases}d^{-n} & \text { if } \varepsilon_{F}-\varepsilon_{\Pi}=0 \\ d^{-2} & \text { if } \varepsilon_{F}-\varepsilon_{\Pi} \neq 0 .\end{cases}
$$

The field $\Pi_{m}(\mathbf{r})$, according to the model discussed at the beginning of Sec. II B, peaks at the colloids and decays far from them, so that it has an approximately rotational symmetry in the range $r_{0}<\rho \ll d$. More precisely, since $\Pi_{m}$ is proportional to $\left|\Pi\left(\left|\rho \mathbf{e}_{\rho}+d \mathbf{e}_{x}\right|\right) \Pi(\rho)\right|^{1 / 2}$, one finds, after expanding in terms of $\rho / d \ll 1$ and in view of Eq. (13),

$$
\Pi_{m}(\mathbf{r}) \sim|\Pi(d) \Pi(\rho)|^{1 / 2}+\mathcal{O}\left(d^{-n / 2-1}\right)
$$

Accordingly, in the leading order in $1 / d$, Eqs. (8a)-(8c) turn into

$$
\begin{aligned}
& \frac{1}{\rho} \frac{\partial}{\partial \rho}\left[\rho \frac{\partial u_{m}}{\partial \rho}\right]+\frac{1}{\rho^{2}} \frac{\partial^{2} u_{m}}{\partial \varphi^{2}} \approx-\frac{2}{\gamma} \Pi_{m}(\rho) \sim d^{-n / 2}, \quad r_{0}<\rho \ll d \\
& {\left[\mathbf{n} \cdot \nabla u_{m}-\frac{u_{m}-\left\langle u_{m}\right\rangle}{r_{0}}\right]_{\rho=r_{0}} \approx\left\{\begin{array}{cc}
\varepsilon_{\Pi_{m}} \sim d^{-n / 2} & \text { if } \varepsilon_{F}-\varepsilon_{\Pi}=0 \\
-\left(r_{0} / 4\right)\left[u^{\prime \prime}(d)-u^{\prime}(d) / d\right] \cos 2 \varphi \sim d^{-2} & \text { if } \varepsilon_{F}-\varepsilon_{\Pi} \neq 0 .
\end{array}\right.}
\end{aligned}
$$

Thus, if $\varepsilon_{F}-\varepsilon_{\Pi}=0$ the near-particle solution is approximately rotationally symmetric and dominated by the pressure field $\Pi_{m}$,

$$
\begin{aligned}
u_{m}(\rho) & \approx A_{0}+r_{0} \varepsilon_{\Pi_{m}} \ln \frac{\rho}{r_{0}}+\frac{2}{\gamma} \int_{r_{0}}^{\rho} d s s \Pi_{m}(s) \ln \frac{s}{\rho} \text { if } \varepsilon_{F}-\varepsilon_{\Pi}=0 \\
& \approx A_{0}+\frac{2}{\gamma} \int_{r_{0}}^{\infty} d s s \Pi_{m}(s) \ln \frac{s}{r_{0}}+\frac{2}{\gamma} \int_{\rho}^{\infty} d s s \Pi_{m}(s) \ln \frac{\rho}{s},
\end{aligned}
$$

where the second expression follows by inserting the estimate $\left[\right.$ Eq. (17)] for $\varepsilon_{\Pi_{m}}$. Only the last term depends on $\rho$, while the second one is an additive constant proportional to $\sqrt{\Pi(d)} \sim d^{-n / 2}$. On the other hand, if $\varepsilon_{F}-\varepsilon_{\Pi} \neq 0$ the nearparticle solution is dominated by the single-particle solution for the boundary condition,

$$
\begin{aligned}
u_{m}(\rho) \approx & A_{0}+\left\{\left[-\frac{r_{0}^{2}}{12}\left(\frac{u^{\prime}(d)}{d}-u^{\prime \prime}(d)\right)+\frac{1}{3} A_{2}\right]\left(\frac{r_{0}}{\rho}\right)^{2}\right. \\
& \left.+A_{2}\left(\frac{\rho}{r_{0}}\right)^{2}\right\} \cos 2 \varphi \text { if } \varepsilon_{F}-\varepsilon_{\Pi} \neq 0
\end{aligned}
$$

The integration constants $A_{0}$ and $A_{2}$ are determined by the solution far from the particles, and for our purposes here, it suffices to provide an estimate of how they depend on the separation $d$. In view of the electrostatic analogy, the solution $u_{m}$ far from the particles, $r \gg d$, can be expressed in terms of a multipolar expansion. The "capillary monopole"

$$
Q_{m}:=\int_{\hat{S}_{\text {men }}} d A 2 \Pi_{m}+\gamma \oint_{\partial S_{1} \cup \partial S_{2}} d \ell \mathbf{n} \cdot\left(-\nabla u_{m}\right)
$$

is found to vanish exactly by virtue of the boundary condition [Eq. (8b)]. The "capillary dipole" vanishes due to the reflection symmetries of the configuration shown in Fig. 2 upon $X \rightarrow-X$ and $Y \rightarrow-Y$. But, in general, the "capillary quadrupole" $D_{m}$ will be nonzero. Therefore, the distant field is ( $C$ is a proportionality constant) 


$$
u_{m}(r \gtrsim d) \sim \frac{\mathrm{d}_{m}}{r^{2}}+C \ln \frac{r}{r_{0}} \int_{r}^{\infty} d s s \Pi_{m}(s),
$$

where we have neglected the angular dependence of the quadrupolar field (we are interested only in the decay with distance) and $\mathrm{d}_{m}$ denotes the typical value of the elements of the quadrupole $\mathrm{D}_{m}$. The second term is approximately the "potential" created by the "capillary charge" beyond $r$, which is not accounted for by the multipolar moments and arises as a correction to the multipolar expansion because the capillary charge $\Pi_{m}$ does not have a compact support. It scales as $r^{2-n} \ln r$. On the other hand, because the capillary monopole of a single particle is of the order of $\gamma r_{0} \varepsilon_{\Pi_{m}}$, the quadrupole of the capillary charge distributed over a region of size $\sim d$ will be $\mathrm{d}_{m} \sim d^{2} \gamma r_{0} \varepsilon_{\Pi_{m}} \sim d^{2-n / 2}$. Thus, the last term is negligible compared to the quadrupolar term if $r \sim d$, provided $n$ $>4$, so that one finally finds $u_{m}(r \sim d) \sim d^{-n / 2}$. For reasons of consistency, we expect that the near-particle solutions in Eqs. (A6a) and (A6b) should scale like this if extrapolated to $\rho$ $\sim d$ : If Eq. (A6a) is evaluated at $\rho \sim d$, one obtains $A_{0}$ $=\mathcal{O}\left(d^{-n / 2}\right)$, while from Eq. (A6b) it follows that $A_{2}=\mathcal{O}\left(d^{-4}\right)$.

In sum, the amplitude of the near-particle solution scales with the interparticle separation as follows:

$$
u_{m}\left(\rho \sim r_{0}\right) \sim\left\{\begin{array}{cc}
d^{-n / 2} & \text { if } \varepsilon_{F}-\varepsilon_{\Pi}=0 \\
d^{-2} & \text { if } \varepsilon_{F}-\varepsilon_{\Pi} \neq 0
\end{array} \quad(n>4) .\right.
$$

\section{APPENDIX B: FLOTATION FORCE AND DISJOINING PRESSURE}

In this appendix, we briefly discuss how our results are modified if the gravitational force is relevant. We also show how the same formal results hold if the lower fluid phase is a thin film on which, instead of gravity, dispersion forces due to a confining substrate are acting, exerting the so-called disjoining pressure.

\section{Flotation force}

The effect of the acceleration of gravity $g$ gives rise to an additional contribution to the free energy [Eq. (2)]: The gravitational potential energy of the fluids with respect to the reference configuration is

$$
\mathcal{F}_{\text {grav }}=\frac{1}{2} \gamma \int_{\hat{S}_{\text {men }}} d A\left(\frac{\hat{u}}{\lambda}\right)^{2},
$$

where we have introduced the capillary length $\lambda$ $:=\sqrt{\gamma /\left[\left(\varrho_{-}-\varrho_{+}\right) g\right]}$ in terms of the mass densities $\varrho_{+}$and $\varrho_{-}$ of the upper and lower fluid phases, respectively. This length has typical values in the millimeter range. A pressure field (force per unit area) can be associated with this free energy,

$$
\hat{\Pi}_{\text {grav }}(\mathbf{r}):=-\frac{\delta \mathcal{F}_{\text {grav }}}{\delta \hat{u}(\mathbf{r})}=-\frac{\gamma}{\lambda^{2}} \hat{u}(\mathbf{r}) .
$$

This expression can be inserted directly into Eq. (3b), and one finds that at large distances from the particles, $\hat{u}(\mathbf{r})$ $\sim \exp (-r / \lambda)$ and the field $\hat{\Pi}_{\text {grav }}(\mathbf{r})$ does indeed decay sufficiently fast. However, one cannot apply the results we have derived previously without certain changes because $\hat{\Pi}_{\text {grav }}$ de-

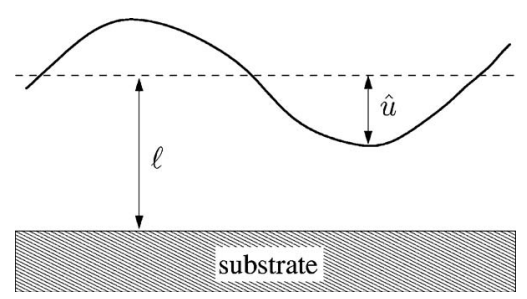

FIG. 9. Schematic drawing of a fluid film on top of a substrate. $\ell$ is the thickness of the film in the reference, flat configuration, and $\hat{u}$ is the deformation of the interface.

pends explicitly on $\hat{u}$. Nevertheless, in the limit of a large capillary length, one can neglect $\mathcal{F}_{\text {grav }}$ (and thus $\hat{\Pi}_{\text {grav }}$ ) altogether and retain only the gravitational force acting directly on the colloidal particle; i.e., $\varepsilon_{\Pi}=0$ and $\hat{F}=F$ is the weight of the colloidal particle (corrected for buoyancy effects). In this case, $V_{\text {men }}(d)$ is given by the superposition approximation [see Eq. (21)] and reproduces the flotation force in the regime $d \ll \lambda .{ }^{18}$

\section{Disjoining pressure}

If the size of the particle lies below the micrometer, gravity is quantitatively negligible. ${ }^{22}$ The same formalism, however, is applicable in the experimentally relevant case that the lower fluid phase is a film of thickness $h(\mathbf{r})=\ell$ $+\hat{u}(\mathbf{r})$ on top of a (solid or liquid) substrate (see Fig. 9). If $\ell$ is within the range of the underlying dispersion forces, an additional contribution $\mathcal{F}_{\text {disp }}$ to the free energy [Eq. (2)] $\operatorname{arises}^{43}$ (neglecting a constant, $\hat{u}$-independent term),

$$
\mathcal{F}_{\text {disp }}=\int_{\hat{S}_{\text {men }}} d A\left[\frac{H}{(\ell+\hat{u})^{2}}+(\ell+\hat{u}) \Delta n \Delta \mu\right],
$$

where $H$ is known as the Hamaker constant, $\Delta n$ is the number density difference between the bulk phases the film and the upper phase belong to, and $\Delta \mu$ is the undersaturation of the upper phase in terms of the chemical potential. The condition that the flat film of thickness $\ell$ is an equilibrium solution imposes the relation $\Delta n \Delta \mu=2 H / \ell^{3}$. The corresponding pressure field associated with $\mathcal{F}_{\text {disp }}$ is called "disjoining pressure,"

$$
\hat{\Pi}_{\text {disp }}(\mathbf{r})=-\frac{\delta \mathcal{F}_{\text {disp }}}{\delta \hat{u}(\mathbf{r})}=\frac{2 H}{(\ell+\hat{u})^{3}}-\frac{2 H}{\ell^{3}} .
$$

In the regime of small deformations, one has $|\hat{u}| \ll \ell$ and $\mathcal{F}_{\text {disp }}$ can be expanded around $\hat{u}=0$, yielding

$$
\mathcal{F}_{\text {disp }} \approx \mathcal{F}_{\text {disp }}[\hat{u}=0]+\frac{1}{2} \gamma \int_{\hat{S}_{\text {men }}} d A\left(\frac{\hat{u}}{\lambda_{\text {disp }}}\right)^{2},
$$

which has the same form as Eq. (B1) with an effective "capillary length" $\lambda_{\text {disp }}=\sqrt{\gamma \ell^{4} /(6 H)}$ [which actually coincides with the so-called lateral correlation length $\xi_{\|}$(Ref. 43)]. Thus, $\hat{\Pi}_{\text {disp }}$ takes the form of Eq. (B2), with $\lambda$ replaced by

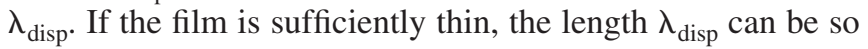
small that it becomes relevant, and a phenomenology may arise which is similar to the macroscopic one induced by gravity. For typical values $H=10^{-20} \mathrm{~J}$ and $\gamma=0.05 \mathrm{~N} / \mathrm{m}$, one has $\lambda_{\text {disp }}=1 \mu \mathrm{m}$ for a film thickness $\ell=0.033 \mu \mathrm{m}$. 


\section{APPENDIX C: EFFECTIVE CAPILLARY POTENTIAL ENERGY IN THE CASE OF AN IDEALLY CONDUCTING FLUID}

In this appendix, we provide the mathematical steps leading to Eqs. (32a) and (32b), which are valid in the limit $d \gg r_{0}$ and from which the effective capillary potential energy is obtained.

In order to compute $\varepsilon_{\Pi}$, we insert the ansatz [Eq. (31)] into the definition [Eq. (15b)]. In the limit $d \rightarrow \infty$, the main contribution to the integral stems from the regions around each particle so that

$$
\varepsilon_{\Pi_{m}} \approx \frac{2}{\gamma r_{0}} \sqrt{\Pi(d)} \int_{r_{0}}^{\infty} d r r \sqrt{\Pi(r)}
$$

With the pressure field given by Eq. (29), this reduces to

$$
\varepsilon_{\Pi_{m}} \approx 2 b(\mu) \varepsilon_{F}\left(\frac{r_{0}}{d}\right)^{3} \int_{1}^{\infty} d x \sqrt{(x-1)^{\mu-1} x^{-(\mu+3)}},
$$

and Eq. (32a) is obtained upon performing the integral (Eq. 3.191.2 in Ref. 38).

For the evaluation of the integral in Eq. (32b), we use the solutions [Eqs. (A1) and (A6a)] valid for $\varepsilon_{F}-\varepsilon_{\Pi_{m}}=0$. With Eq. (29), this leads to

$$
\begin{aligned}
\nabla u & =\frac{\mathbf{e}_{r}}{\gamma r} \int_{r}^{\infty} d s s \Pi(s) \\
& =\mathbf{e}_{r} b(\mu) \varepsilon_{F} \frac{r_{0}}{r} \int_{r / r_{0}}^{\infty} d x(x-1)^{\mu-1} x^{-(\mu+4)} \\
& =\frac{1}{4} \mathbf{e}_{r} b(\mu) \varepsilon_{F}\left(\frac{r_{0}}{r}\right)^{5}{ }_{2} F_{1}\left(1-\mu, 4 ; 5 ; \frac{r_{0}}{r}\right)
\end{aligned}
$$

and, with the ansatz [Eq. (31)], to

$$
\begin{aligned}
\nabla u_{m} & \approx \frac{2 \mathbf{e}_{r}}{\gamma r} \int_{r}^{\infty} d s s \sqrt{\Pi(d) \Pi(s)} \\
& =2 \mathbf{e}_{r} b(\mu) \varepsilon_{F} \frac{r_{0}}{r}\left(\frac{r_{0}}{d}\right)^{3} \int_{r / r_{0}}^{\infty} d x(x-1)^{(\mu-1) / 2} x^{-(\mu+3) / 2} \\
& =2 \mathbf{e}_{r} b(\mu) \varepsilon_{F}\left(\frac{r_{0}}{d}\right)^{3}\left(\frac{r_{0}}{r}\right)_{2}^{2} F_{1}\left(\frac{1-\mu}{2}, 1 ; 2 ; \frac{r_{0}}{r}\right) .
\end{aligned}
$$

[Concerning the last lines in Eqs. (C3) and (C4), see Eq. 3.194.2 in Ref. 38.] This enables one to obtain

$$
\begin{aligned}
& \int_{S_{\mathrm{men}, 2}} d A\left(\nabla u_{m}\right) \cdot\left(\nabla u_{2}\right) \\
& \approx \pi b(\mu)^{2} \varepsilon_{F}^{2}\left(\frac{r_{0}}{d}\right)^{3} \int_{r_{0}}^{\infty} d r r\left(\frac{r_{0}}{r}\right)^{7} \\
& \quad \times{ }_{2} F_{1}\left(1-\mu, 4 ; 5 ; \frac{r_{0}}{r}\right){ }_{2} F_{1}\left(\frac{1-\mu}{2}, 1 ; 2 ; \frac{r_{0}}{r}\right)
\end{aligned}
$$

so that Eqs. (32b) and (33) follow upon a change of variable in the integral.

\section{APPENDIX D: ASYMPTOTIC BEHAVIOR OF THE ELECTROSTATIC POTENTIAL AND OF THE ELECTRIC FIELD}

In this appendix, we discuss the asymptotic behaviors of the functions $\mathcal{I}_{n}(k)$ defined in Eqs. (43a)-(43c). First, we mention that at first sight the integrals may appear to be divergent due to the weak power law decay of the integrands, which are, however, oscillatory. Actually, the integrals are regularized by an exponential, so that, for instance,

$$
\mathcal{I}_{a}(k):=\lim _{h \rightarrow 0} \int_{0}^{\infty} d x \mathrm{e}^{-h x} J_{0}(x) \frac{x}{\left(\epsilon_{1} / \epsilon_{2}\right) x+\sqrt{x^{2}+k^{2}}},
$$

reflecting the physical situation that the charge $q$ is positioned at a (dimensionless) height $h>0$ above the flat interface. In the following mathematical manipulations, this regularization scheme is implied, which, unless required, we do not write explicitly to avoid a clumsy notation.

Reference 4 provides a method to obtain the expansion of $\mathcal{I}_{a}(k)$ in powers of $\epsilon_{1} / \epsilon_{2} \ll 1$. The idea is to split the integrals into a sum of two terms involving only odd or even powers of the ratio $\epsilon_{1} / \epsilon_{2}$, respectively. This leads to ${ }^{44}$

$$
\begin{aligned}
\mathcal{I}_{a}(k)= & \mathrm{e}^{-k}+\frac{\epsilon_{1}}{\epsilon_{2}}\left[\frac{\pi}{2} k\left(I_{0}(k)-\mathbf{L}_{0}(k)\right)-1\right] \\
& +\mathcal{O}\left(\epsilon_{1} / \epsilon_{2}\right)^{2}, \quad k \ll\left(\epsilon_{2} / \epsilon_{1}\right)^{2}
\end{aligned}
$$

in terms of the Bessel function $I_{0}$ and the Struve function $\mathbf{L}_{0} \cdot{ }^{38}$ We note that the validity of this expression is restricted to sufficiently small values of $k$. However, for the typical values of the ratio $\epsilon_{2} / \epsilon_{1}$ occurring in the experiments so far, this does not impose any physically relevant constraint on $k$. The asymptotic behavior for large $k$ is

$$
\mathcal{I}_{a}(k) \approx \mathrm{e}^{-k}+\frac{\epsilon_{1}}{\epsilon_{2}} \frac{1}{k^{2}}, \quad 1 \ll k \ll\left(\epsilon_{2} / \epsilon_{1}\right)^{2} .
$$

As a general result, ${ }^{4}$ the coefficients of the odd powers of $\epsilon_{1} / \epsilon_{2}$ decay algebraically for $k \gg 1$, while the coefficients of the even powers decay exponentially. With Eq. (43c), this leads to

$$
\mathcal{I}_{c}(k) \approx k \mathrm{e}^{-k}+3 \frac{\epsilon_{1}}{\epsilon_{2}} \frac{1}{k^{2}}, \quad 1 \ll k \ll\left(\epsilon_{2} / \epsilon_{1}\right)^{2} .
$$

The procedure employed in Ref. 4 can be extended to analyze $\mathcal{I}_{b}(k)$. The radical is eliminated from the denominator of the integrand in Eq. (43b), and the integral is split as follows:

$$
\begin{aligned}
\mathcal{I}_{b}(k)= & \frac{\epsilon_{2}}{\epsilon_{1}} \int_{0}^{\infty} d x J_{0}(x) \frac{x\left(x^{2}+k^{2}\right)}{\left[1-\left(\epsilon_{1} / \epsilon_{2}\right)^{2}\right] x^{2}+k^{2}} \\
& -\int_{0}^{\infty} d x J_{0}(x) \frac{x^{2} \sqrt{x^{2}+k^{2}}}{\left[1-\left(\epsilon_{1} / \epsilon_{2}\right)^{2}\right] x^{2}+k^{2}} .
\end{aligned}
$$

The first term involves only odd powers of $\epsilon_{1} / \epsilon_{2}$, the second term only even powers. The first integral can be rewritten as 


$$
\begin{aligned}
\frac{\epsilon_{2}}{\epsilon_{1}} \int_{0}^{\infty} & d x J_{0}(x) \frac{x\left(x^{2}+k^{2}\right)}{\left[1-\left(\epsilon_{1} / \epsilon_{2}\right)^{2}\right] x^{2}+k^{2}} \\
= & \frac{\epsilon_{2} / \epsilon_{1}}{1-\left(\epsilon_{1} / \epsilon_{2}\right)^{2}} \int_{0}^{\infty} d x J_{0}(x) x \\
& \times\left[1+\frac{k^{2}-k^{2} /\left[1-\left(\epsilon_{1} / \epsilon_{2}\right)^{2}\right]}{x^{2}+k^{2} /\left[1-\left(\epsilon_{1} / \epsilon_{2}\right)^{2}\right]}\right] .
\end{aligned}
$$

With the identities (see Eqs. 6.623.2 and 6.532.4 in Ref. 38)

$$
\int_{0}^{\infty} d x J_{0}(x) x=0
$$

and

$$
\int_{0}^{\infty} d x J_{0}(x) \frac{x}{x^{2}+a^{2}}=K_{0}(a)
$$

the first integral in Eq. (D5) can be written as

$$
\begin{aligned}
& \frac{\epsilon_{2}}{\epsilon_{1}} \int_{0}^{\infty} d x J_{0}(x) \frac{x\left(x^{2}+k^{2}\right)}{\left[1-\left(\epsilon_{1} / \epsilon_{2}\right)^{2}\right] x^{2}+k^{2}} \\
& \quad=-\frac{\epsilon_{1} / \epsilon_{2}}{\left[1-\left(\epsilon_{1} / \epsilon_{2}\right)^{2}\right]^{2}} k^{2} K_{0}\left(k / \sqrt{1-\left(\epsilon_{1} / \epsilon_{2}\right)^{2}}\right) \\
& \quad=-\left(\epsilon_{1} / \epsilon_{2}\right) k^{2} K_{0}(k)+\mathcal{O}\left(\epsilon_{1} / \epsilon_{2}\right)^{3} .
\end{aligned}
$$

The second integral in Eq. (D5) can be written to the leading order in $\epsilon_{1} / \epsilon_{2}$ as

$$
\begin{aligned}
\int_{0}^{\infty} & d x J_{0}(x) \frac{x^{2} \sqrt{x^{2}+k^{2}}}{x^{2}+k^{2}} \\
& =\int_{0}^{\infty} d x J_{0}(x)\left[\sqrt{x^{2}+k^{2}}-\frac{k^{2}}{\sqrt{x^{2}+k^{2}}}\right] .
\end{aligned}
$$

We introduce the exponential regularization as $\exp ($ $-h \sqrt{x^{2}+k^{2}}$ ) and note the identity (Eq. 6.637.1 in Ref. 38)

$$
\begin{aligned}
\int_{0}^{\infty} d x J_{0}(x) \frac{\mathrm{e}^{-h \sqrt{x^{2}+k^{2}}}}{\sqrt{x^{2}+k^{2}}}= & I_{0}\left(\frac{k}{2}\left[\sqrt{h^{2}+1}-h\right]\right) \\
& \times K_{0}\left(\frac{k}{2}\left[\sqrt{h^{2}+1}+h\right]\right) .
\end{aligned}
$$

Therefore, the right-hand side of Eq. (D10) can be rewritten as

$$
\begin{aligned}
\int_{0}^{\infty} & d x J_{0}(x) \frac{x^{2} \sqrt{x^{2}+k^{2}}}{x^{2}+k^{2}} \\
= & \lim _{h \rightarrow 0}\left[\frac{\partial^{2}}{\partial h^{2}}-k^{2}\right] \int_{0}^{\infty} d x J_{0}(x) \frac{\mathrm{e}^{-h \sqrt{x^{2}+k^{2}}}}{\sqrt{x^{2}+k^{2}}} \\
= & \frac{1}{2} k^{2}\left[I_{1}\left(\frac{k}{2}\right) K_{1}\left(\frac{k}{2}\right)-I_{0}\left(\frac{k}{2}\right) K_{0}\left(\frac{k}{2}\right)\right] \\
= & k \frac{d}{d k}\left[k I_{0}\left(\frac{k}{2}\right) K_{1}\left(\frac{k}{2}\right)\right] .
\end{aligned}
$$

Therefore, from Eqs. (D9) and (D12) one finally arrives at the following expansion:

$$
\begin{aligned}
\mathcal{I}_{b}(k)= & \frac{1}{2} k^{2}\left[I_{0}\left(\frac{k}{2}\right) K_{0}\left(\frac{k}{2}\right)-I_{1}\left(\frac{k}{2}\right) K_{1}\left(\frac{k}{2}\right)\right]-\frac{\epsilon_{1}}{\epsilon_{2}} k^{2} K_{0}(k) \\
& +\mathcal{O}\left(\epsilon_{1} / \epsilon_{2}\right)^{3} .
\end{aligned}
$$

This expansion may also be restricted to sufficiently small values of $k$, although we have not found this upper bound as function of $\epsilon_{1} / \epsilon_{2}$. However, as argued above, this possible constraint is expected to be physically irrelevant because, typically, $\epsilon_{1} / \epsilon_{2}$ is sufficiently small. The asymptotic behavior of this expression for $k \gg 1$ is

$$
\mathcal{I}_{b}(k) \approx \frac{1}{k}-\sqrt{\frac{\pi}{2}} \frac{\epsilon_{1}}{\epsilon_{2}} k^{3 / 2} \mathrm{e}^{-k}, \quad 1 \ll k, \epsilon_{2} / \epsilon_{1} .
$$

${ }^{1}$ P. Pieranski, Phys. Rev. Lett. 45, 569 (1980).

${ }^{2}$ J. Joannopoulos, Nature (London) 414, 257 (2001).

${ }^{3}$ A. Dinsmore, M. F. Hsu, M. Nikolaides, M. Márquez, A. Bausch, and D. Weitz, Science 298, 1006 (2002).

${ }^{4}$ A. J. Hurd, J. Phys. A 18, L1055 (1985).

${ }^{5}$ R. Aveyard, J. H. Clint, D. Nees, and V. N. Paunov, Langmuir 16, 1969 (2000).

${ }^{6}$ J. Ruiz-García, R. Gámez-Corrales, and B. I. Ivlev, Physica A 236, 97 (1997).

${ }^{7}$ F. Ghezzi and J. Earnshaw, J. Phys.: Condens. Matter 9, L517 (1997).

${ }^{8}$ D. Stamou, C. Duschl, and D. Johannsmann, Phys. Rev. E 62, 5263 (2000).

${ }^{9}$ M. Quesada-Pérez, A. Moncho-Jordá, F. Martínez-López, and R. Hidalgo-Alvarez, J. Chem. Phys. 115, 10897 (2001).

${ }^{10}$ F. Ghezzi, J. C. Earnshaw, M. Finnis, and M. McCluney, J. Colloid Interface Sci. 238, 433 (2001).

${ }^{11}$ S. J. Mejía-Rosales, A. Gil-Villegas, B. I. Ivlev, and J. Ruiz-García, J. Phys.: Condens. Matter 14, 4795 (2002).

${ }^{12}$ M. G. Nikolaides, A. R. Bausch, M. F. Hsu, A. D. Dinsmore, M. P. Brenner, C. Gay, and D. A. Weitz, Nature (London) 420, 299 (2002).

${ }^{13}$ G. Tolnai, A. Agod, M. Kabai-Faix, A. L. Kovács, J. J. Ramsden, and Z. Hórvölgyi, J. Phys. Chem. B 107, 11109 (2003).

${ }^{14}$ O. Gómez-Guzmán and J. Ruiz-García, J. Colloid Interface Sci. 291, 1 (2005).

${ }^{15}$ W. Chen, S. Tan, Z. Huang, T.-K. Ng, W. T. Ford, and P. Tong, Phys. Rev. E 74, 021406 (2006).

${ }^{16}$ J. C. Fernández-Toledano, A. Moncho-Jordá, F. Martínez-López, and R. Hidalgo-Alvarez, Langmuir 20, 6977 (2004).

${ }^{17}$ M. M. Nicolson, Proc. Cambridge Philos. Soc. 45, 288 (1949).

${ }^{18}$ D. Y. C. Chan, J. D. Henry, Jr., and L. R. White, J. Colloid Interface Sci. 79, 410 (1981)

${ }^{19}$ K. D. Danov, P. A. Kralchevsky, and M. P. Boneva, Langmuir 20, 6139 (2004).

${ }^{20}$ M. Megens and J. Aizenberg, Nature (London) 424, 1014 (2003).

${ }^{21}$ L. Foret and A. Würger, Phys. Rev. Lett. 92, 058302 (2004).

${ }^{22}$ M. Oettel, A. Domínguez, and S. Dietrich, Phys. Rev. E 71, 051401 (2005).

${ }^{23}$ A. Domínguez, M. Oettel, and S. Dietrich, J. Phys.: Condens. Matter 17, S3387 (2005).

${ }^{24}$ M. Oettel, A. Domínguez, and S. Dietrich, Langmuir 22, 846 (2006).

${ }^{25}$ M. Oettel, A. Domínguez, and S. Dietrich, J. Phys.: Condens. Matter 17, L337 (2005).

${ }^{26}$ A. Würger and L. Foret, J. Phys. Chem. B 109, 16435 (2005).

${ }^{27}$ A. Domínguez, M. Oettel, and S. Dietrich, arXiv:cond-mat/0611329.

${ }^{28}$ A. Würger, Europhys. Lett. 75, 978 (2006).

${ }^{29}$ A. Domínguez, M. Oettel, and S. Dietrich, Europhys. Lett. 77, 68002 (2007).

${ }^{30}$ In this respect, Ref. 22 is incomplete, but the same conclusion about the irrelevance of this effect is reached. Reference 28 contains several important mathematical errors (Ref. 29), and the corresponding conclusion erroneously disagrees with the actual irrelevance of the finite-size effect.

${ }^{31}$ In actual experiments, there is a certain degree of polydispersity in size, shape, electric charge, etc., which we do not expect to alter the conclusions qualitatively if it remains small enough.

${ }^{32}$ Which fluid phase is to be the top one depends on the experimental setup. Usually gravity breaks the up-down symmetry. 
${ }^{33}$ This seems to be actually a reliable approximation for the experimental setups considered so far in the literature, but see the discussion in Sec. IV

${ }^{34}$ R. Aveyard, B. P. Binks, J. H. Clint, P. D. I. Fletcher, T. S. Horozov, B. Neumann, V. N. Paunov, J. Annesley, S. W. Botchway, D. Nees, A. W Parker, A. D. Ward, and A. N. Burgess, Phys. Rev. Lett. 88, 246102 (2002).

${ }^{35}$ Due to the rapid decay of $\hat{\Pi}$ far from the particles, in the limit $L \rightarrow \infty$, the length scale $L$ enters into the problem only if $\varepsilon_{\Pi} \neq \varepsilon_{F}$ and in that case just as an upper bound on $d$ to avoid a logarithmic divergence [see the discussion after Eq. (A1)].

${ }^{36}$ Note that by symmetry $\left\langle u_{2}\right\rangle_{2}=\left\langle u_{1}\right\rangle_{1}$ and $\left\langle u_{m}\right\rangle_{2}=\left\langle u_{m}\right\rangle_{1}$.

${ }^{37}$ K. D. Danov and P. A. Kralchevsky, J. Colloid Interface Sci. 298, 213 (2006).

${ }^{38}$ I. S. Gradshteyn and I. M. Ryzhik, Table of Integrals, Series, and Products, 5th ed. (Academic, London, 1994).
${ }^{39}$ Next-to-leading terms in the expansion require a more elaborate calculation than the simple Taylor expansion, which yields undefined expressions for them.

${ }^{40}$ M. Oettel, A. Domínguez, M. Tasinkevych, and S. Dietrich, preprint, 2007.

${ }^{41}$ K. D. Danov, P. A. Kralchevsky, and M. P. Boneva, Langmuir 22, 2653 (2006); this work is de facto a correction (Ref. 24) of previous work (Ref. 19).

${ }^{42}$ D. Frydel, S. Dietrich, and M. Oettel, Phys. Rev. Lett. 99, 118302 (2007).

${ }^{43}$ S. Dietrich, in Phase Transitions and Critical Phenomena, edited by C. Domb and J. L. Lebowitz (Academic, London, 1988), Vol. 12, p. 1.

${ }^{44}$ We note (i) a misprint in Eq. (10) in Ref. 4 because there the term $2^{m}$ should be in the numerator and (ii) that the series provided in that Eq. (10) can actually be carried out and is proportional to the error function. 